\title{
Chiral condensates for neutron stars in hadron-quark crossover: from a parity doublet nucleon model to an NJL quark model
}

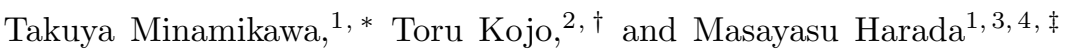 \\ ${ }^{1}$ Department of Physics, Nagoya University, Nagoya 464-8602, Japan \\ ${ }^{2}$ Key Laboratory of Quark and Lepton Physics (MOE) and Institute of Particle Physics, \\ Central China Normal University, Wuhan 430079, China \\ ${ }^{3}$ Kobayashi-Maskawa Institute for the Origin of Particles and the Universe, Nagoya University, Nagoya 464-8602, Japan \\ ${ }^{4}$ Advanced Science Research Center, Japan Atomic Energy Agency, Tokai 319-1195, Japan
}

(Dated: December 13, 2021)

\begin{abstract}
We study the chiral condensates in neutron star matter from nuclear to quark matter domain. We describe nuclear matter with a parity doublet model (PDM), quark matter with the NambuJona-Lasino (NJL) model, and a matter at the intermediate density by interpolating nuclear and quark matter equations of state. The model parameters are constrained by nuclear physics and neutron star observations. Various condensates in the interpolated domain are estimated from the chemical potential dependence of the condensates at the boundaries of the interpolation. The use of the PDM with substantial chiral invariant mass $\left(m_{0} \gtrsim 500 \mathrm{MeV}\right.$, which is favored by the neutron star observations) predicts the mild chiral restoration, and the significant chiral condensate remains to baryon density $n_{B} \sim 2-3 n_{0}\left(n_{0} \simeq 0.16 \mathrm{fm}^{-3}\right.$ : nuclear saturation density), smoothly approaching the NJL predictions for the color-flavor-locked phase at $n_{B} \gtrsim 5 n_{0}$. The same method is applied to estimate diquark condensates, number densities of up-, down- and strange-quarks, and the lepton fraction. In our descriptions the chiral restoration in the interpolated domain proceeds with two conceptually distinct chiral restoration effects; the first is associated with the positive scalar density in a nucleon, relevant in dilute regime, and the other primarily arises from the modification of the quark Dirac sea, which is triggered by the growth of the quark Fermi sea. We discuss several qualitative conjectures to interpolate the microphysics in nuclear and quark matter.
\end{abstract}

\section{INTRODUCTION}

The chiral condensates are important quantities to characterize the state of matter in Quantum Chromodynamics (QCD). While its high temperature behavior at low baryon density has been studied in the lattice Monte Carlo simulations [1-3] its behavior at low temperature and high baryon density remains unknown due to the lack of reliable first principle computations.

In this situation recent neutron star (NS) observations, which tightly constrain the equations of state (EOS) of matter, are useful tools to access the properties of dense matter (see, e.g., Ref. 4 for a review and Ref. [5] for a mini-review). The existence of twosolar mass $\left(2 M_{\odot}\right)$ NSs with $1.91 M_{\odot}$ [6 8], 2.01 $M_{\odot}$ [9], and $M=2.08 \pm 0.07 M_{\odot}$ [10], the radius constraint $R_{1.4} \lesssim 13 \mathrm{~km}$ for $1.4 M_{\odot}$ NS deduced from the gravitational event GW170817 11, and recent NICER constraints on $R_{1.4} \simeq R_{2.08} \simeq 12-13 \mathrm{~km} \mathrm{[1214}$, disfavor the strong first order phase transitions for the domain between the nuclear saturation density $\left(n_{0} \simeq 0.16 \mathrm{fm}^{-3}\right)$ and the core density achieved in the $2 M_{\odot}$ NSs. The core baryon density of $2 M_{\odot}$ NS is $n_{B} \gtrsim 4-5 n_{0}$, presumably high enough to apply quark matter descriptions, so we infer that nuclear matter smoothly transforms into quark matter (modulo weak first order phase transitions).

\footnotetext{
* minamikawa@hken.phys.nagoya-u.ac.jp

$\dagger$ torujj@mail.ccnu.edu.cn

$\ddagger$ harada@hken.phys.nagoya-u.ac.jp
}

Following the three-window picture developed in Refs. 15 17, we have been constructing series of unified EOS covering from nuclear to quark matter, and used the NS observations to constrain them [18 21]. We use a nuclear model at $n_{B} \lesssim 2 n_{0}$, a quark model at $n_{B} \gtrsim 5 n_{0}$, and then interpolate them to construct EOS for $n_{B} \simeq 2-5 n_{0}$. The resulting EOS can be made consistent with available NS constraints by choosing the proper range of parameters in our model. Meanwhile, the real strength of our approach is that, with the microscopic degrees of freedom being manifest, we can use the model to predict physical quantities other than EOS and get some insights on the microphysics [4].

The previous works based on interpolation schemes 15 21, however, did not manifestly address the microscopic quantities in the interpolated domain, and thus did not utilize the full potential of the framework. In this paper, we examine various condensates and matter compositions by constructing unified generating functionals for the pressure, including external fields coupled to condensates. The condensates are obtained by differentiating the functionals with respect to the external fields. In this way it is possible to examine the chiral and diquark condensates as well as lepton fractions from nuclear to quark matter domain. The condensates in the interpolated domain are affected by the physics of nuclear and quark matter through the boundary conditions for the interpolation. We examine condensates in nuclear and quark matter models in detail, and then infer the physics relevant in the interpolated domain.

In this work, a nuclear matter is described by a parity 
double model (PDM) for nucleons [22, 23] coupled to the meson potentials. (For nuclear matter descriptions based on the PDM, see e.g. Refs. 24 51]). In the PDM, nucleons with positive and negative parities form a doublet in which the masses are split by the chiral condensates; in chiral symmetric phase the masses get degenerated with the finite chiral invariant mass, $m_{0}$. Finite temperature lattice simulations indicated the parity degeneracy of nucleons at finite mass [52, supporting the idea of the chiral invariant mass. With a larger $m_{0}$, the nucleon mass can reach the experimental value within weak couplings between nucleons and the chiral condensates; in turn the chiral condensates become less sensitive to changes in the nuclear medium, and hence the chiral restoration driven by the increase of density proceeds more mildly. This feature also affects the structure of neutron stars. In the context of the $\sigma-\omega-\rho$ type models [53 55] for neutron star matter, the reduced $\sigma N$ coupling leads to a less attractive $\sigma$ exchange, so that a weaker repulsion by the $\omega$ exchange is sufficient to reproduce the physics near the saturation density. This trends becomes more important at higher baryon density where the $\sigma$ fields decrease but $\omega$ fields increase. In the context of NS observations, the reduction of $\omega$-repulsion reduces NS radii from $13-14$ $\mathrm{km}$ to $12-13 \mathrm{~km}$ [21].

A quark matter is described by the standard NambuJona-Lasinio (NJL) model [56] plus additional effective interactions, the vector repulsion and diquark attraction [18. The vector repulsion is particularly important in the context of the chiral restoration, as it tempers the growth in density, smoothing out the chiral restoration [57, 58. Meanwhile the inclusion of diquark terms leads to the color-flavor-locked (CFL) phase in which $u d-, d s-$, and $s u$-diquark pairs condense, and these pairings favor a larger quark Fermi sea [59. After including these two competing effects, we found that the substantial amount of the chiral condensates remain in quark matter, and the effective quark masses at $n_{B} \sim 5 n_{0}$ for up- and downquarks are $\sim 50 \mathrm{MeV}$, for strange quarks $\sim 300 \mathrm{MeV}$ [4.

The chiral condensates in the interpolated domain, which are influenced by matching to nuclear and quark matter models, are supposed to contain the mixture of two distinct chiral restoration effects. The first type is the chiral restoration due to the positive scalar charge in a nucleon; the sign is opposite to the negative vacuum chiral density so that these charges cancel in spatial average, leading to the reduction of chiral condensates. This sort of chiral restoration does not necessarily demands the knowledges about the structural changes of hadrons in medium, and the estimate is valid at least in dilute regime. Meanwhile, the NJL quark models describe more fundamental changes associated with changes in the Dirac sea or in the constituent quark properties [4. Implementing such information through the high density boundary conditions introduces a qualitative trend that differs from the high density extrapolation of the PDM.

The recipe to calculate chiral condensates in the inter- polated domain is also usable for other condensates, such as diquark condensates and quark densities for various flavors. In particular the strangeness and lepton fractions have distinct trends from those in purely hadronic models.

This paper is structured as follows. In Sec II, we discuss the PDM as a nuclear matter model. The equations of state and chiral condensates for light $(u, d)$ and strange quarks are discussed. In Sec III] we discuss the NJL model with effective diquark and vector interactions. In Sec IV] we discuss the recipe to compute condensates in the interpolated domain, and examine the behaviors of various condensates. In Sec $\mathrm{V}$, we give several conjectures to describe our results in the microphysics language. Sec VI is devoted to the summary.

In this paper we write the integrals over space, $\int_{x}=$ $\int \mathrm{d}^{4} x, \int_{\mathbf{x}}=\int \mathrm{d}^{3} \mathbf{x}$, and over momentum, $\int_{\mathbf{p}}=\int \frac{\mathrm{d}^{3} \mathbf{p}}{(2 \pi)^{3}}$.

\section{NUCLEAR MATTER}

\section{A. Parity Doublet Model (PDM)}

The mean field grand potential functional in our PDM consists of the meson and baryon contributions [21,

$$
\Omega_{\mathrm{bare}}\left(\mu_{B}, \mu_{Q} ; \varphi\right)=\Omega_{M}(\varphi)+\Omega_{B}\left(\mu_{B}, \mu_{Q} ; \varphi\right),
$$

where $\varphi$ collectively denotes the meson fields $(\sigma, \omega, \rho)$, and $\mu_{B}$ and $\mu_{Q}$ are the baryon number and the electrical charge chemical potential, respectively. The meson part is

$$
\begin{aligned}
\Omega_{M}= & -\frac{1}{2} \bar{\mu}^{2} \sigma^{2}+\frac{1}{4} \lambda_{4} \sigma^{4}-\frac{1}{6} \lambda_{6} \sigma^{6}-m_{\pi}^{2} f_{\pi} \sigma \\
& -\frac{1}{2} m_{\omega}^{2} \omega^{2}-\frac{1}{2} m_{\rho}^{2} \rho^{2},
\end{aligned}
$$

where $m_{\pi}$ and $f_{\pi}$ are the pion mass and decay constant in vacuum, respectively, and the baryon part is $(\Theta(x)$ is the step function)

$$
\Omega_{B}=-2 \sum_{i= \pm} \sum_{\alpha=p, n} \int_{\mathbf{p}}\left(\mu_{\alpha}^{*}-E_{\mathbf{p}}^{i}\right) \Theta\left(\mu_{\alpha}^{*}-E_{\mathbf{p}}^{i}\right)
$$

where the index $i= \pm$ is for the parities of nucleons and $\alpha$ for protons and neutrons. Here we used the no-sea approximation which neglects the Dirac sea contributions from nucleons. The single particle energies for nucleons and chemical potentials are respectively given by

$$
\begin{aligned}
E_{\mathbf{p}}^{i} & =\sqrt{\mathbf{p}^{2}+m_{i}^{2}}, \\
\mu_{p}^{*} & =\mu_{B}-g_{\omega N N} \omega-\frac{1}{2} g_{\rho N N} \rho+\mu_{Q}, \\
\mu_{n}^{*} & =\mu_{B}-g_{\omega N N} \omega+\frac{1}{2} g_{\rho N N} \rho .
\end{aligned}
$$

In the PDM, there are doublet fields $N_{1}$ and $N_{2}$ that couple to the $\sigma$ with the couplings $g_{1}$ and $g_{2}$. By calculating the eigenstates for coupled equations of $N_{1}$ and 
TABLE I: Physical inputs in vacuum in unit of $\mathrm{MeV}$.

\begin{tabular}{cccccc}
\hline \hline$m_{\pi}$ & $f_{\pi}$ & $m_{\omega}$ & $m_{\rho}$ & $m_{+}\left(=m_{N}\right)$ & $m_{-}$ \\
\hline 140 & 92.4 & 783 & 776 & 939 & 1535 \\
\hline \hline
\end{tabular}

$N_{2}$, the masses for positive and negative parity nucleons are given by the following form,

$$
m_{ \pm} \equiv \sqrt{m_{0}^{2}+\left(\frac{g_{1}+g_{2}}{2}\right)^{2} \sigma^{2}} \mp \frac{\left|g_{1}-g_{2}\right|}{2} \sigma,
$$

where $m_{0}$ is the chiral invariant mass. Actually $m_{-}$does not appear for $n_{B} \leq 2 n_{0}$ in our choices of $m_{0}$, so we will use the notation $m_{N}$ for the positive parity nucleon mass $m_{+} ; m_{N} \equiv m_{+}$. The parameters used in our analyses and the output are summarized in Tables.I. II] and III.

We minimize the functional $\Omega_{\text {bare }}$ at given $\mu_{B}$ and $\mu_{Q}$ by varying fields $\varphi$, and write the solutions $\varphi_{*}\left(\mu_{B}, \mu_{Q}\right)$. The substituting $\varphi_{*}$ into the functional, and normalize it by subtracting the vacuum contributions, our thermodynamic potential for the hadronic part is given by

$$
\Omega_{h}\left(\mu_{B}, \mu_{Q}\right) \equiv \Omega_{\text {bare }}\left(\mu_{B}, \mu_{Q} ; \varphi_{*}\right)-\Omega_{\text {bare }}\left(0,0 ; \varphi_{\text {vac }}\right),(7)
$$

where we have chosen the model parameters in such a way that $\varphi_{\text {vac }}=\left.\left(\sigma_{*}, \omega_{*}, \rho_{*}\right)\right|_{\mu_{B}=\mu_{Q}=0}=\left(f_{\pi}, 0,0\right)$. In the following we will drop off the subscript $*$ to simplify the notation, unless otherwise stated.

Finally we impose the charge neutrality and $\beta$ equilibrium condition. The lepton part is

$$
\Omega_{\text {lepton }}=-2 \sum_{l=e, \mu} \int_{\mathbf{p}}\left(\mu_{l}-E_{\mathbf{p}}^{l}\right) \Theta\left(\mu_{l}-E_{\mathbf{p}}^{l}\right),
$$

where $\mu_{l}=-\mu_{Q}$, common for electrons and muons, and $E_{\mathbf{p}}^{l}=\sqrt{m_{l}^{2}+\mathbf{p}^{2}}$ with $m_{e}=0.5 \mathrm{MeV}$ and $m_{\mu}=105.7$ $\mathrm{MeV}$. For a given $\mu_{B}$, we tune $\mu_{Q}$ to $\mu_{Q}^{*}$ that satisfies the neutrality condition,

$$
\left.\frac{\partial\left(\Omega_{h}+\Omega_{\text {lepton }}\right)}{\partial \mu_{Q}}\right|_{\mu_{Q}^{*}}=0 .
$$

In the following, whenever we mention EOS at a given $\mu_{B}$, we will implicitly choose $\mu_{Q}=\mu_{Q}^{*}\left(\mu_{B}\right)$, so that EOS depends on $\mu_{B}$ only.

\section{B. Chiral condensate in the PDM}

To calculate the chiral condensate in the PDM, we differentiate the thermodynamic potential with respect to the current quark mass. In our model the explicit chiral symmetry breaking enters only through the term $-m_{\pi}^{2} f_{\pi} \sigma$; we neglect the mass dependence in the other coupling constants in front of higher powers in meson fields. Such couplings are supposed to appear in the form of $\sim\left(\varphi / M_{q}\right)^{n}$ with the effective quark mass $M_{q} \simeq 300$ $\mathrm{MeV}$ from the integration of quarks. Hence we expect
TABLE II: Values of model parameters determined for several choices of $m_{0}$. The values of the slope parameter $L_{0}$ is also shown as output.

\begin{tabular}{c|ccccc}
\hline \hline$m_{0}[\mathrm{MeV}]$ & 500 & 600 & 700 & 800 & 900 \\
\hline$g_{1}$ & 9.02 & 8.48 & 7.81 & 6.99 & 5.96 \\
$g_{2}$ & 15.5 & 14.9 & 14.3 & 13.4 & 12.4 \\
$\bar{\mu}^{2} / f_{\pi}^{2}$ & 22.7 & 22.4 & 19.3 & 11.9 & 1.50 \\
$\lambda_{4}$ & 41.9 & 40.4 & 35.5 & 23.1 & 4.43 \\
$\lambda_{6} f_{\pi}^{2}$ & 16.9 & 15.8 & 13.9 & 8.89 & 0.636 \\
$g_{\omega N N}$ & 11.3 & 9.13 & 7.30 & 5.66 & 3.52 \\
$g_{\rho N N}$ & 7.31 & 7.86 & 8.13 & 8.30 & 8.43 \\
\hline$L_{0}[\mathrm{MeV}]$ & 93.76 & 86.24 & 83.04 & 81.33 & 80.08 \\
\hline \hline
\end{tabular}

TABLE III: Saturation properties used to determine the model parameters: the saturation density $n_{0}$, the binding energy $B_{0}$, the incompressibility $K_{0}$ and the symmetry energy $S_{0}$.

\begin{tabular}{cccc}
\hline \hline$n_{0}\left[\mathrm{fm}^{-3}\right]$ & $B_{0}[\mathrm{MeV}]$ & $K_{0}[\mathrm{MeV}]$ & $S_{0}[\mathrm{MeV}]$ \\
\hline 0.16 & 16 & 240 & 31 \\
\hline \hline
\end{tabular}

that those couplings depend mainly on the distance scale shorter than the physics of pions, and hence the current quark mass is expected to be a small perturbation in powers of $\sim\left(m_{q} / M_{q}\right)^{n}$. Using the Gell-Mann-OakesRenner relation, the explicit symmetry breaking term can be written as

$$
\Omega_{\mathrm{ESB}}=-m_{\pi}^{2} f_{\pi} \sigma=m_{q}\langle(\bar{u} u+\bar{d} d)\rangle_{0} \frac{\sigma}{f_{\pi}},
$$

where $m_{q}=\left(m_{u}+m_{d}\right) / 2$ is the average of current quark masses of up and down quarks and $\langle(\bar{u} u+\bar{d} d)\rangle_{0}$ is the chiral condensate in vacuum. Accordingly

$$
\langle(\bar{u} u+\bar{d} d)\rangle \equiv \frac{\partial \Omega_{\mathrm{ESB}}}{\partial m_{q}}=\langle(\bar{u} u+\bar{d} d)\rangle_{0} \frac{\sigma}{f_{\pi}},
$$

is the in-medium chiral condensate in our PDM (here we neglected $m_{q}$ dependence of $\langle(\bar{u} u+\bar{d} d)\rangle_{0}$ which is higher orders in $\left.m_{q} / M_{q}\right)$. Below we focus on how $\sigma$ changes as baryon density increases.

\section{Chiral scalar density in a nucleon}

For the estimate of in-medium chiral condensates, it is useful to use the scalar charge in a nucleon, $N_{\sigma}$. It can be related to the nucleon mass in vacuum as

$$
\begin{aligned}
N_{\sigma} & =\int_{\mathbf{x}}\langle N|(\bar{u} u+\bar{d} d)(x)| N\rangle \\
& =\left\langle N\left|\frac{\partial H_{\mathrm{QCD}}}{\partial m_{q}}\right| N\right\rangle=\frac{\partial m_{N}^{\mathrm{vac}}}{\partial m_{q}},
\end{aligned}
$$

where $H_{\mathrm{QCD}}$ is the QCD hamiltonian, and in the last step we used the Hellmann-Feynman theorem [60]. 
In the PDM, the nucleon mass depends on the current quark mass only through the modification of $\sigma$, so we calculate the scalar charge at vacuum as

$$
N_{\sigma}=\frac{\partial m_{N}^{\mathrm{vac}}}{\partial m_{q}}=\frac{\partial \sigma^{\mathrm{vac}}}{\partial m_{q}}\left(\frac{\partial m_{N}}{\partial \sigma}\right)_{\sigma=\sigma_{\mathrm{vac}}} .
$$

When we calculate the variation of $\sigma^{\mathrm{vac}}$, we prepare the thermodynamic potentials at various $m_{q}$, calculate $\sigma^{\text {vac }}$ for each potential, and evaluate the impact of the mass variation on $\sigma^{\mathrm{vac}}$. The mass derivative of $\sigma^{\mathrm{vac}}$ is proportional to the static correlator ${ }^{1} \sim\left\langle(\sigma-\langle\sigma\rangle)^{2}\right\rangle \sim m_{\sigma}^{-2}$, so is bigger for a smaller scalar meson mass.

Multiplying $m_{q}$ to the scalar charge, we get the socalled nucleon sigma term,

$$
\Sigma_{N}=m_{q} N_{\sigma}=\int_{\vec{x}}\left\langle N\left|m_{q}(\bar{u} u+\bar{d} d)\right| N\right\rangle,
$$

in which the combination $m_{q}(\bar{u} u+\bar{d} d)$ is renormalization group invariant. The scalar density is often discussed in this form as it is more directly related to the experimental quantities. The traditional estimate [61] is $\Sigma_{N} \simeq 45 \mathrm{MeV}$ (which includes up- plus down-quark contributions), but currently the estimates based on lattice QCD or combined analyses of the lattice QCD and the chiral perturbation theory range over $40-70 \mathrm{MeV}$. (see a review 60. and the references therein.) Substituting $m_{q} \simeq 5 \mathrm{MeV}$, we get $N_{\sigma} \simeq 8-14$, so the scalar density is

$$
\frac{N_{\sigma}}{4 \pi R_{N}^{3} / 3}=\left((0.24-0.30) \mathrm{GeV} \frac{1 \mathrm{fm}}{R_{N}}\right)^{3},
$$

where $R_{N}$ is the size of a nucleon, which is $\sim 1 \mathrm{fm}$. This is roughly the same order of magnitude as the vacuum scalar density but with the opposite sign. The scalar isoscalar radius is $\left\langle r_{s}^{2}\right\rangle \simeq(0.7-1.2 \mathrm{fm})^{2}$ 62]. For comparison, the values in the PDM for various $m_{0}$ are listed in Table IV

We note that our $m_{\sigma} \simeq 270-410 \mathrm{MeV}$ is smaller than the mass of the scalar meson $f_{0}(500)$ (with the width $\sim 500 \mathrm{MeV}){ }^{2}$ while $\Sigma_{N}$ in our model is larger than the traditional value $\simeq 45 \mathrm{MeV}$. Recalling that $\Sigma_{N} \propto \partial \sigma^{\mathrm{vac}} / m_{q} \sim m_{\sigma}^{-2}$, better agreement with empirical values may be achieved by improving our model to accommodate a larger $m_{\sigma}$. This can be done by, e.g., adding higher order polynomials of $\sigma$ fields to modify

1 The mass derivative of the quark condensate is related to the (connected) scalar correlator at zero momentum,

$$
\begin{aligned}
\frac{\partial\langle\bar{q} q(x)\rangle}{\partial m_{q}} & \sim \int \mathcal{D} q \mathcal{D} \bar{q}[\bar{q} q(x)] \frac{\partial}{\partial m_{q}}\left(\mathrm{e}^{-\int_{x^{\prime}} m_{q} \bar{q} q\left(x^{\prime}\right)+\ldots} / Z\right) \\
& \sim \int_{x^{\prime}}\left\langle[\bar{q} q(x)]\left[\bar{q} q\left(x^{\prime}\right)\right]\right\rangle_{\text {conn. }} \sim \lim _{q \rightarrow 0} \frac{1}{q^{2}+m_{\sigma}^{2}}
\end{aligned}
$$

2 It is not a trivial issue whether one should interpret $\sigma$ in mean field models as the physical scalar meson. the curvature of the effective potential. Such a fine tuning is beyond the scope of this paper; we keep using the parameter set used for analyses of EOS in Ref.[21, and shall not redo EOS construction. We expect that overall qualitative trends of chiral restoration can be studied within the present setup.

The relation $N_{\sigma}=\partial m_{N}^{\text {vac }} / \partial m_{q}$ tells us that the scalar charge should be positive, as the nucleon mass is supposed to increase with the current quark masses. Since the chiral condensate in vacuum is negative, the nucleon scalar charges tend to cancel the vacuum chiral condensate.

\section{Dilute regime}

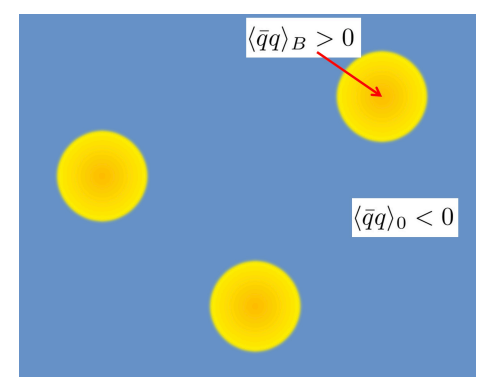

FIG. 1: Chiral condensates in dilute regime. The region dominated by the vacuum chiral condensate has the negative scalar charge while in nucleons the scalar charges are positive.

In the dilute regime (see Fig 1) we can make a solid statement; here nucleons are widely separated, so the scalar density in medium is estimated by simply adding the scalar charges of nucleons to the vacuum condensate, and then taking the spatial average, i.e.,

$$
\langle(\bar{u} u+\bar{d} d)\rangle \simeq\langle(\bar{u} u+\bar{d} d)\rangle_{0}+n_{B} N_{\sigma}
$$

or equivalently one can write

$$
\sigma \simeq f_{\pi}\left(1+\frac{n_{B} N_{\sigma}}{\langle(\bar{u} u+\bar{d} d)\rangle_{0}}\right)
$$

This is the famous linear density approximation, which implies that the value of $\sigma$ decreases as $n_{B}$ increases.

The violation of this approximation signals the end of the dilute regime. Figure2 shows the ratio of the quark condensate, $\langle\bar{u} u\rangle /\langle\bar{u} u\rangle_{0}=\sigma / f_{\pi}$, versus the neutron number density $n_{n}$ in pure neutron matter and compare it with the linear density approximation. Our mean field results have milder chiral restoration than in the linear density approximation. Similar trends have been found in the analyses based on the chiral effective theories including the fluctuations of pions 6365

We stress that, in light of the PDM, the chiral restoration discussed here does not necessarily have the immediate impacts on the properties of baryons nor the Dirac 
TABLE IV: Values of PDM in vacuum.

\begin{tabular}{c|ccccc}
\hline \hline$m_{0}[\mathrm{MeV}]$ & 500 & 600 & 700 & 800 & 900 \\
\hline$\left(\partial m_{N} / \partial \sigma\right)_{\text {vac. }}$ & 7.97 & 7.01 & 5.87 & 4.56 & 3.07 \\
$m_{\sigma}[\mathrm{MeV}]$ & 396 & 414 & 388 & 332 & 271 \\
$\Sigma_{N}[\mathrm{MeV}]$ & 91.9 & 74.0 & 70.5 & 74.6 & 75.7 \\
\hline \hline
\end{tabular}

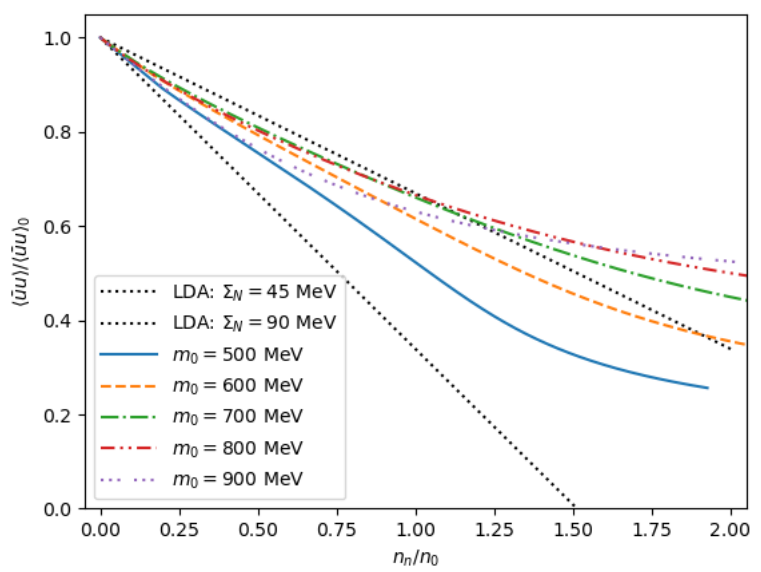

FIG. 2: Ratio of the quark condensate in the PDM $\langle\bar{u} u\rangle /\langle\bar{u} u\rangle_{0}=\sigma / f_{\pi}$ versus the neutron number density $n_{n}$.

sea. These considerations are consistent with our no sea approximation for the thermodynamic potential for nucleons (see, Eq.(3p) and modest changes in nucleon mass in the PDM. We also note that, in a high temperature transition from a hadron resonance gas (HRG) to a quark gluon plasma (QGP), the chiral condensates substantially decrease just below the transition temperature, but at such temperature the HRG model with the vacuum hadron masses still work well to quantitatively describe the lattice data [66, 67. We will address this point again when we discuss the chiral restoration in quark matter where the modifications in the Dirac sea are likely.

\section{E. Strange quark condensate}

The increase in nuclear density also affects the strange quark condensate, as nucleons contain the sea strange quarks. The abundance of sea strange quarks in a nucleon can be estimated by using the strangeness sigmaterm,

$$
\Sigma_{s N}=m_{s} \frac{\partial m_{N}}{\partial m_{s}}=\int_{\vec{x}}\left\langle N\left|m_{s} \bar{s} s\right| N\right\rangle=m_{s} N_{s} .
$$

Our PDM does not manifestly include the strangeness so that we simply substitute the vacuum value for the linear density approximation,

$$
\langle\bar{s} s\rangle \simeq\langle\bar{s} s\rangle_{0}+n_{B} N_{s} .
$$

This estimate will be used when we consider the interpolation between nuclear and quark matter.
Our estimate of the strange quark condensate contains several errors even in dilute regime. In fact, the strangeness content in a nucleon is difficult to determine, as it is a small effect not related to the valence quarks. The estimates from the lattice QCD range from $\simeq 17$ $\mathrm{MeV}$ to $\simeq 42 \mathrm{MeV}$. Taking $m_{s} \simeq 100 \mathrm{MeV}$, we estimate $N_{s} \simeq 0.2-0.4$, which is about $\sim(0.01-0.05) N_{\sigma}$.

The positivity of strangeness sigma-term found on the lattice implies that $\partial m_{N} / \partial m_{s}$ is positive. This is by no means trivial, as strange quarks are not valence quarks in a nucleon, and increasing strange quark does not readily increase the valence quark mass nor the nucleon mass. One possible way to express $\partial m_{N} / \partial m_{s}>0$ is to assume the validity of the expression as in Eq. 13],

$$
N_{s}=\frac{\partial m_{N}^{\mathrm{vac}}}{\partial m_{s}} \simeq \frac{\partial \sigma^{\mathrm{vac}}}{\partial m_{s}}\left(\frac{\partial m_{N}}{\partial \sigma}\right)_{\sigma=\sigma_{\mathrm{vac}}},
$$

[reminder: $\sigma$ is made of the sum of up- and downquarks, not including strange quarks] and to consider the Kobayashi-Maskawa-'tHooft term for the $U(1)_{A}$ anomaly,

$$
\mathcal{L}_{\mathrm{KMT}} \sim C(\bar{u} u)(\bar{d} d)(\bar{s} s)+\cdots,
$$

where we wrote only the product of scalar densities. A larger strange quark more explicitly breaks the chiral symmetry and hence enhances the size of strange quark condensate in vacuum. Within mean field treatments (as in the NJL model), a larger strange condensate contributes to the term like

$$
\mathcal{L}_{\mathrm{KMT}} \sim C\langle\bar{s} s\rangle_{0}\left[\langle\bar{d} d\rangle_{0} \times \bar{u} u+\langle\bar{u} u\rangle_{0} \times \bar{d} d\right]+\cdots
$$

which, for $C<0$, assists the chiral symmetry breaking and increases the effective quark masses for up- and down-quarks. These relations suggest that a larger $m_{s}$ firstly enhances $\langle\bar{s} s\rangle_{0}$, and then the effective masses of up- and down-quarks through the anomaly term. Within this description, $m_{N}$ or $\sigma_{\text {vac }}$ increases for a larger $m_{s}$, and hence $N_{s}>0$ follows.

\section{QUARK MATTER (CFL PHASE)}

\section{A. Overall picture}

We use the NJL model for our quark matter descriptions. The model does not describe the confinement, but explains the hadron phenomenology not very sensitive to the confining effects [56], e.g., the low energy constants for a hadronic effective Lagrangian for energies $\lesssim 1 \mathrm{GeV}$. The NJL model is supposed to capture the physics at semi-hard scale, $0.2 \mathrm{GeV} \lesssim p \lesssim 1 \mathrm{GeV}$, the scale between confinement and chiral symmetry breaking [68, 69. In terms of the distance scale, those quark models capture the physics in a hadron, $\sim 1 \mathrm{fm}$, but do not resolve the partonic structure of constituent quarks [70]. We expect the NJL type constituent quark models to give reasonable descriptions on the bulk quantities at density where baryons begin to overlap. 
Our NJL model includes the vector repulsion and diquark attraction in addition to the standard NJL model [56]. These additional interactions are motivated by the fact that the successful hadron description needs not only the use of proper constituent quark masses, but also the color interactions at semi-hard scale. For instance the color magnetic interactions are needed to account for the level splitting such as $N-\Delta$ or $\pi-\rho$ [71]. We try to include the attractive part of such interactions in our diquark terms. At high density they lead to the diquark condensation, and, in three-flavor model, the colorflavor-locked (CFL) pairing. Meanwhile the magnetic interactions in repulsive channels can be used to explain the channel dependence in baryon-baryon interactions at short distance [72, 73, e.g., hard core repulsion between nucleons. Our vector repulsion can be thought of the parameterization of such short distance repulsion.

While the vector and diquark interactions contribute to the energy density with the opposite signs, their effects do not cancel much as the density dependence are different. The vector repulsion acts on the whole bulk part of the quark Fermi sea, contributing $\varepsilon_{\text {vector }} \sim+n_{B}^{2}$, while the diquark attraction mainly affects on quarks near the Fermi surface, contributing $\varepsilon_{\text {diquark }} \sim-\Delta^{2} n_{B}^{2 / 3}$. Both contributions can stiffen equations of state [18].

\section{B. NJL model}

Our NJL Lagrangian is 18

$$
\mathcal{L}=\mathcal{L}_{\mathrm{NJL}}+\mathcal{L}_{\mathrm{V}}+\mathcal{L}_{\mathrm{d}} .
$$

The first term is the standard three-flavor NJL model with the anomaly term $\left(\lambda_{A}\right.$ and $\tau_{A}(A=0, \cdots 8)$ being the identity element $(A=0)$ and Gell-Mann matrices $(A=1, \cdots, 8)$ in color and flavor spaces, respectively, normalized as $\left.\operatorname{tr}\left[\lambda_{A} \lambda_{B}\right]=\operatorname{tr}\left[\tau_{A} \tau_{B}\right]=2 \delta_{A B}\right)$,

$$
\begin{aligned}
\mathcal{L}_{\mathrm{NJL}} & =\bar{q}\left(i \not \partial-\hat{m}_{q}+\hat{\mu} \gamma_{0}\right) q \\
& +G \sum_{A=0}^{8}\left[\left(\bar{q} \tau_{A} q\right)^{2}+\left(\bar{q} i \gamma_{5} \tau_{A} q\right)^{2}\right] \\
& -K\left[\operatorname{det}_{f} \bar{q}\left(1-\gamma_{5}\right) q+\operatorname{det}_{f} \bar{q}\left(1+\gamma_{5}\right) q\right],
\end{aligned}
$$

where $\hat{m}_{q}=$ diag. $\left(m_{u}, m_{d}, m_{s}\right)$ is the current quark mass matrix for $u, d, s$-quarks, and $\hat{\mu}$ is

$$
\hat{\mu}=\mu_{B} / 3+\mu_{c 3} \lambda_{3}+\mu_{c 8} \lambda_{8}+\mu_{Q} Q,
$$

with $\mu_{c 3}, \mu_{c 8}, \mu_{Q}$ being the third and eighth components of color charges, and the electric charges; the charge matrix is $Q=$ diag. $(2 / 3,-1 / 3,-1 / 3)$. For the values of the coupling constants $(G, K)$ and the UV cutoff of the model $\Lambda_{\mathrm{NJL}}$, we chose the values of Hatsuda-Kunihiro parameters: $G \Lambda_{\mathrm{NJL}}^{2}=1.835, K \Lambda_{\mathrm{NJL}}^{5}=9.29$ with $\Lambda_{\mathrm{NJL}}=631.4$ $\mathrm{MeV}$. This set of parameters successfully describes the low energy hadron physics in vacuum, such as masses of Nambu-Goldstone bosons, decay constants, and so on.
The vector and diquark interactions are

$$
\begin{aligned}
\mathcal{L}_{\mathrm{V}}=-g_{V}\left(\bar{q} \gamma^{\mu} q\right)\left(\bar{q} \gamma_{\mu} q\right), \\
\mathcal{L}_{\mathrm{d}}=H \sum_{A, B=2,5,7}\left[\left(\bar{q} \tau_{A} \lambda_{B} C \bar{q}^{t}\right)\left(q^{t} C \tau_{A} \lambda_{B} q\right)\right. \\
\left.\quad+\left(\bar{q} i \gamma_{5} \tau_{A} \lambda_{B} C \bar{q}^{t}\right)\left(q^{t} C i \gamma_{5} \tau_{A} \lambda_{B} q\right)\right],
\end{aligned}
$$

where $C=i \gamma_{0} \gamma_{2}$ is the charge conjugation matrix. In mean field approaches, the coupling constants $\left(g_{V}, H\right)$ are not well constrained in vacuum as the density and diquark mean fields (see below) are vanishing. But the impacts of these couplings are very large at finite baryon density, and the range of the couplings has been constrained by neutron star observables [20, 21, 74] and by a model of non-perturbative gluon exchanges [75].

The chiral and diquark condensates ( $\tilde{\sigma}$ slightly differs from the $\sigma$ in the PDM by a factor)

$$
\tilde{\sigma}_{f}=\left\langle\bar{q}_{f} q_{f}\right\rangle, \quad d_{a}=\left\langle q^{t} C \gamma_{5} R_{a} q\right\rangle,
$$

where the indices $f=1,2,3$ correspond to $u, d, s$-flavors, $\left(R_{1}, R_{2}, R_{3}\right)=\left(\tau_{7} \lambda_{7}, \tau_{5} \lambda_{5}, \tau_{2} \lambda_{2}\right)$. The quark density $n_{q}\left(=N_{c} n_{B}\right)$ is the sum of $u, d, s$-quark densities

$$
n_{q}=\sum_{f} n_{f}, \quad n_{f}=\left\langle q_{f}^{\dagger} q_{f}\right\rangle
$$

Within the mean field approximation, the single particle energies are obtained by diagonalizing the matrix

$$
S^{-1}(k)=\left(\begin{array}{cc}
\not k-\hat{M}+\gamma^{0} \hat{\mu}_{\mathrm{MF}} & \gamma_{5} \Delta_{i} R_{i} \\
-\gamma_{5} \Delta_{i}^{*} R_{i} & \not k-\hat{M}-\gamma^{0} \hat{\mu}_{\mathrm{MF}}
\end{array}\right)
$$

whose eigenvalues, $\epsilon_{i=1, . ., 72}$ (among which 18 eigenvalues are independent), give the single particle (sp) energies of quarks. The effective chiral mass, diquark gap, and effective chemical potential are

$$
\begin{aligned}
M_{i} & =m_{i}-4 G \sigma_{i}+K\left|\epsilon_{i j k}\right| \sigma_{j} \sigma_{k}, \\
\Delta_{i} & =-2 H d_{i}, \\
\hat{\mu}_{\mathrm{MF}} & =\mu_{B} / 3-2 g_{V} n_{q}+\mu_{3} \lambda_{3}+\mu_{8} \lambda_{8}+\mu_{Q} Q .
\end{aligned}
$$

Here $-2 g_{V} n_{q}$ is from the mean field repulsion. The $\mu_{3}$ and $\mu_{8}$ will be tuned to enforce the color-neutrality constraints, $\partial \Omega / \partial \mu_{3,8}=0$.

With the single particle energies and condensation terms, the thermodynamic functional is given by $\Omega[\phi]=$ $\Omega_{\mathrm{sp}}[\phi]+\Omega_{\text {cond }}[\phi]+\Omega_{\text {lepton }}$, where the quark part is

$$
\begin{aligned}
\Omega_{\mathrm{sp}}[\phi] & =-2 \sum_{i=1}^{18} \int^{\Lambda} \frac{d^{3} \mathbf{p}}{(2 \pi)^{3}} \frac{\epsilon_{i}}{2}, \\
\Omega_{\mathrm{cond}}[\phi] & =\sum_{i}\left(2 G \sigma_{i}^{2}+H d_{i}^{2}\right)-4 K \sigma_{u} \sigma_{d} \sigma_{s}-g_{V} n_{q}^{2}(36)
\end{aligned}
$$

and the lepton part is treated in the same way as in the PDM (Eq. (8)); the value of $\mu_{Q}$ is tuned to satisfy the charge neutrality condition. The $\phi$ in quark part collectively describes $(\sigma, d)$. After minimizing $\Omega[\phi]$ by $\phi=\phi_{*}$ under the neutrality constraints, we obtain the thermodynamic potential $\Omega=\Omega\left[\phi_{*}\right]$. 


\section{Chiral condensates in the CFL quark matter}

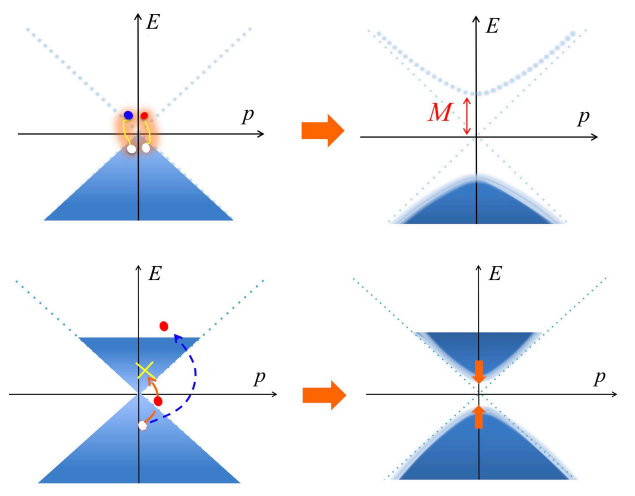

FIG. 3: The chiral symmetry breaking by condensation of quark-antiquark pairs; (upper) in vacuum; (lower) in medium. In the latter the pairing is blocked by the quark Fermi sea.

In quark matter, the quark Fermi sea disfavors the quark-antiquark pairing which triggers the formation of the chiral condensates (Fig 3 ). This is because having an antiquark costs the energy of the order of the quark Fermi energy; regarding an antiquark as a hole in the Dirac sea, its creation needs to bring the particle in the Dirac sea to the domain beyond the Fermi sea, avoiding the Pauli blocking. Hence, as density increases, the chiral condensates naturally dissociate. Alternatively, the particle-particle or particle-hole pairings are favored as they involve only the degrees of freedom near the Fermi surface [76].

It is important to note that the chiral restoration discussed here differs from what we have described in dilute nuclear matter; there the chiral restoration is due to the cancellation between positive and negative charges. But the positive scalar charge in each nucleon nor the negative scalar charges from the vacuum do not change much with density; only the sum does. Meanwhile, in quark matter we do consider the reduction in each of positive and negative scalar charges; both the Dirac sea and the constituent quark masses change. In the context of the PDM, the Dirac sea modification should be responsible for the reduction of the chiral invariant mass $m_{0}$.

\section{CONDENSATES IN A UNIFIED EOS}

In this section we construct a unified EOS and condensates by interpolating the nuclear and quark models in the previous sections. We compute the chiral and diquark condensates, and the composition of matter with $(u, d, s)$-quarks and leptons (electrons and muons, $e, \mu)$.

\section{A. Unified generating functional}

In order to compute a unified EOS and a condensate $\phi$, we first construct a generating functional $P\left(\mu_{B} ; J\right)$ in which $J$ is the external field coupled to the condensate $\phi$. After computing the generating functional, one can differentiate it with respect to $J$,

$$
\phi=-\left.\frac{\partial P}{\partial J}\right|_{J=0},
$$

to calculate the condensate at a given $\mu_{B}$.

The generating functional for the nuclear domain, $n_{B} \leq 2 n_{0}$, is given by the PDM, and for the quark matter domain, $n_{B} \geq 5 n_{0}$, by the NJL model. These functionals are interpolated by requiring the continuity up to the second derivatives at the boundaries, $2 n_{0}$ and $5 n_{0}$. As an interpolating function we use a polynomial function with six constants $a_{n}(J)$,

$$
P_{\mathrm{I}}\left(\mu_{B} ; J\right)=\sum_{n=0}^{5} a_{n}(J) \mu_{B}^{n}
$$

for the intermediate region $2 n_{0}<n_{B}<5 n_{0}$. We write the chemical potentials at the boundaries as $\mu_{B}^{L}$ and $\mu_{B}^{U}$ which satsify

$$
n_{B}\left(\mu_{B}^{L} ; J\right)=2 n_{0}, \quad n_{B}\left(\mu_{B}^{U} ; J\right)=5 n_{0} .
$$

It is important to remember that, as we use the fixed densities for the boundaries, $\mu_{B}^{L}$ and $\mu_{B}^{U}$ depend on $J$. The six parameters $a_{n}$ are determined from the six boundary conditions,

$$
\left.\frac{\partial^{k} P_{\mathrm{I}}}{\left(\partial \mu_{B}\right)^{k}}\right|_{\mu_{B}^{L}\left(\mu_{B}^{U}\right)}=\left.\frac{\partial^{k} P_{\mathrm{PDM}(\mathrm{NJL})}}{\left(\partial \mu_{B}\right)^{k}}\right|_{\mu_{B}^{L}\left(\mu_{B}^{U}\right)},
$$

where $k=0,1,2$. Determination of $a_{n}$ at $J=0$ gives us the unified EOS.

The model parameters of the generating functional are restricted by the causality condition,

$$
c_{s}^{2}=\frac{\mathrm{d} P}{\mathrm{~d} \varepsilon}=\frac{n_{B}}{\mu_{B} \chi_{B}} \leq 1
$$

where $c_{s}$ is the sound velocity and $\chi_{B}=\partial^{2} P / \partial \mu_{B}^{2}$ is the baryon number susceptibility.

\section{B. A practical method to compute condensates}

In this subsection, we explain a practical method to compute condensates from our generating functional. In this method, it is not necessary to manifestly compute $P\left(\mu_{B}, J\right)$ for various $J$, but we utilize only the $\mu_{B^{-}}$ dependence of the condensate at $J=0$ for each interpolating boundary. This method is useful especially when we need to compute many condensates. 
For our interpolating function, the expression of condensate $\phi$ in the interpolated domain is given by

$$
\phi_{\mathrm{I}}=-\left.\frac{\partial P_{\mathrm{I}}}{\partial J}\right|_{J=0}=-\left.\sum_{n=0}^{5} \frac{\partial a_{n}}{\partial J}\right|_{J=0} \mu_{B}^{n} .
$$

Thus the determination of $\phi_{\mathrm{I}}$ is equivalent to the determination of six constants $\partial a_{n} / \partial J(n=0, \ldots, 5)$ at $J=0$. Recalling that our matching condition at a given $J$ is given in Eq. 40, we obtain the $\partial a_{n} / \partial J$ by taking the $\mu_{B}$-derivatives of Eq. 40,

$$
\frac{\partial}{\partial J}\left(\left.\frac{\partial^{k} P_{\mathrm{I}}}{\left(\partial \mu_{B}\right)^{k}}\right|_{\mu_{B}^{L}\left(\mu_{B}^{U}\right)}\right)=\frac{\partial}{\partial J}\left(\left.\frac{\partial^{k} P_{\mathrm{PDM}(\mathrm{NJL})}}{\left(\partial \mu_{B}\right)^{k}}\right|_{\mu_{B}^{L}\left(\mu_{B}^{U}\right)}\right),
$$

where $k=0,1,2$. We set $J=0$ in the end. When we compute the derivatives, we should remember that $\mu_{B}^{L}\left(\mu_{B}^{U}\right)$ depend on $J$, see Appendix. A for the details. Importantly, all these derivatives at $J=0$ can be obtained using only quantities at a given $\mu_{B}$ and $J=0$; it is not necessary to refer to the quantities for various $J$ nor $\mu_{B}$; our computations are local. Hence our method reduces computational efforts significantly.

\section{Numerical results}

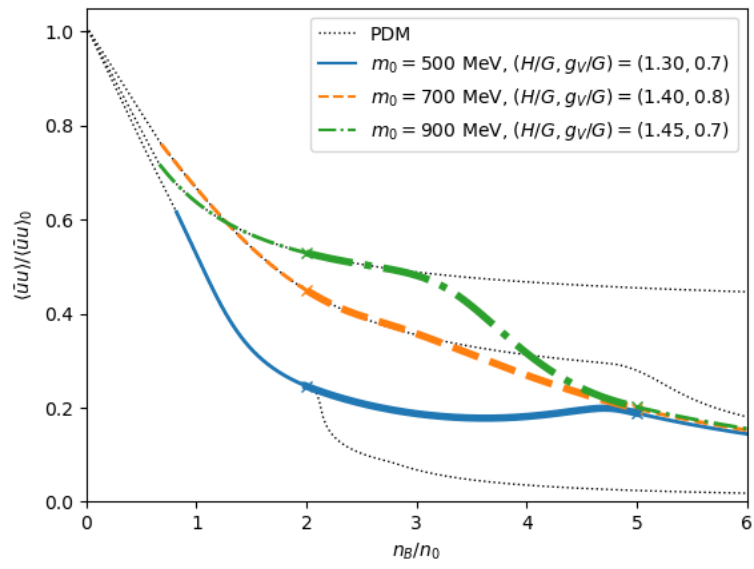

FIG. 4: Density dependence of the ratio of the chiral condensates.

Using the method presented in the last section, we compute the light quark chiral condensate $\langle(\bar{u} u+\bar{d} d)\rangle$, the strange quark condensate $\langle\bar{s} s\rangle$, the diquark gaps $\Delta_{i}$, and the quark number densities $n_{f}$, from nuclear to quark matter domain. Unless otherwise stated we pick up three sample parameter sets for $\left(m_{0}[\mathrm{MeV}], H / G, g_{V} / G\right)$ as

$$
\begin{array}{ll}
(500,1.30,0.7), & \text { [blue solid] } \\
(700,1.40,0.8), & \text { [orange dashed] } \\
(900,1.45,0.7), & \text { [green dash dotted] }
\end{array}
$$

all of which lead to EOS with the causal speed of sound. Here [...] indicates the types of lines used in figures for these parameters. As a guide, we will also show the extrapolation of the PDM results by black dotted lines.

\section{Light quark chiral condensates}

Figure4 shows the ratio of the chiral condensate in medium to the vacuum counterpart, $\langle(\bar{u} u+\bar{d} d)\rangle /\langle(\bar{u} u+\bar{d} d)\rangle_{0}$. It is clear that the condensate at the boundaries put strong bias to the condensate in the interpolated domain.

The PDM results are sensitive to the choice of $m_{0}$. For $m_{0}=500 \mathrm{MeV}$, the chiral condensate reduces radically, as the $N-\sigma$ coupling must be strong to reproduce $m_{N}=939 \mathrm{MeV}$, and hence changes in nuclear medium have large impacts on the behavior of $\sigma$. For a larger $m_{0}$, the medium effects on $\sigma$ are smaller. Meanwhile the NJL model typically leads to the chiral condensates whose magnitudes are $20-30 \%$ of the vacuum values.

As we have mentioned in SecIID the chiral restoration within the PDM may underestimate or neglect the chiral restoration at the quark level. At higher density the effects of interactions among nucleons are stronger, and it is natural to consider structural changes of nucleons and the modifications of $m_{0}$ and the couplings. The bias from the quark matter side is used to infer the trends of these effects. Therefore, although phenomenological, we believe that our interpolating method offers reasonably balanced descriptions.

\section{Strange chiral condensates}

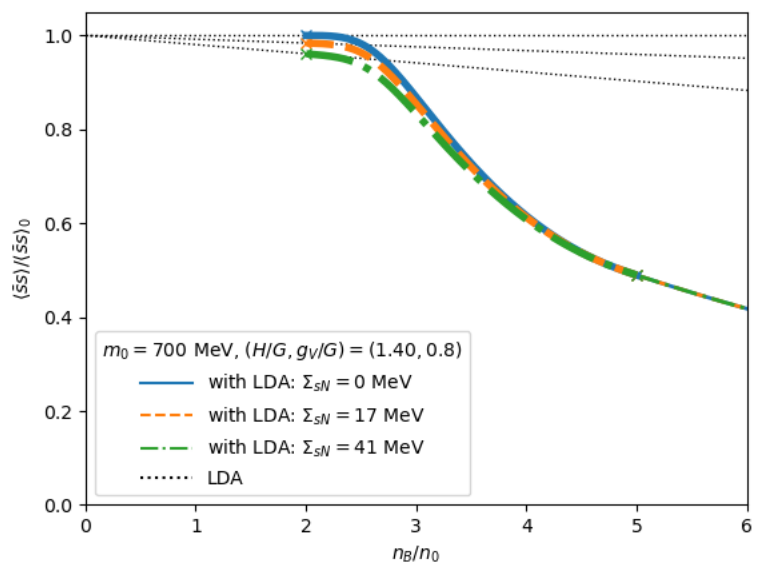

FIG. 5: Density dependence of the strange quark condensate normalized by the vacuum counterpart.

Another quantity of interest is the strange quark condensate, as shown in Fig.5. Let us first look at the trend at $n_{B} \leq 2 n_{0}$. Although the strange quark contribu- 
tions are not defined in the PDM of this work, we estimate them by assuming the linear density approximation Eq.20 with the strangeness sigma-term. For the sigma-term we use $\Sigma_{s N}=0,17,41 \mathrm{MeV}$. For $\Sigma_{s N}=0$, the strange quark condensate is density independent, while the values $\Sigma_{s N}=17,41 \mathrm{MeV}$ are picked up from Ref. 60. We fixed the parameters $m_{0}=700 \mathrm{MeV}$ and $\left(H, g_{V}\right) / G=(1.4,0.8)$, and then vary the values of $\Sigma_{s N}$. As expected, Fig 5 shows that the chiral restoration in the strange quark sector is very small in the nuclear domain.

Beyond $2 n_{0}$, the strange quark condensate starts to reduce, and the reduction from the vacuum value is $\sim 20 \%$ at $n_{B} \simeq 3 n_{0}$. By construction, this reduction is due to the bias from the boundary condition in the quark matter side. In the quark matter at $n_{B} \simeq 5 n_{0}$, the reduction is $\simeq 50 \%$. This chiral restoration should mainly come from two effects. One is the suppression of the anomaly term, $\sim\langle\bar{u} u\rangle\langle\bar{d} d\rangle(\bar{s} s)$, associated with the reduction of light quark condensates. The other is due to the appearance of the strange quark Fermi sea. In our model the strangeness density begins to share $\sim 10 \%$ of quark density at $n_{B} \simeq 3 n_{0}$, where we suppose that the strange quark sector is modified at the quark level.

\section{Diquark gaps and number density}

Figure6 shows the numerical result of the interpolated diquark gaps in the $u d$-pairing channel (upper panel) and $d s$-pairing channel (lower panel) for the same choice of parameters in Fig 4. The diquark condensates are assumed to be zero at $n_{B} \leq 2 n_{0}$. In the CFL quark matter, the isospin symmetry holds in very good accuracy, while in nuclear matter the pairing is zero, so $\Delta_{d s} \simeq \Delta_{u s}$ hold in good accuracy for whole domain.

Next we examine the correlation between the diquark condensates and quark number density shown in Fig.7. In the nuclear domain, the quark density for each flavor is calculated from the proton and neutron densities $\left(n_{p}\right.$ and $\left.n_{n}\right)$ as $n_{u}=2 n_{p}+n_{n}, n_{d}=n_{p}+2 n_{n}$, and $n_{s}=0$. We note that the growth of the diquark condensates is strongly correlated with the growth of quark number density, as can be seen from the comparison of Figs. 6 and 7. This is qualitatively reasonable; a larger Fermi surface allows more diquark pairs, and the associated energy reduction of the system in turn assists the growth in density. Another important effect is on the flavor asymmetry. In particular, the number density of the strange quark in the nuclear matter is zero, while the strong pairing between the strange quark and the other light quarks favor the equal population of $u, d, s$-quarks in the quark matter. As a result the number density of the strange quark rapidly increases in the interpolated region and the onset of the strangeness takes place at lower density than without the pairings. This competition between the mass asymmetry and the pairing effects determines the fraction of each quark.
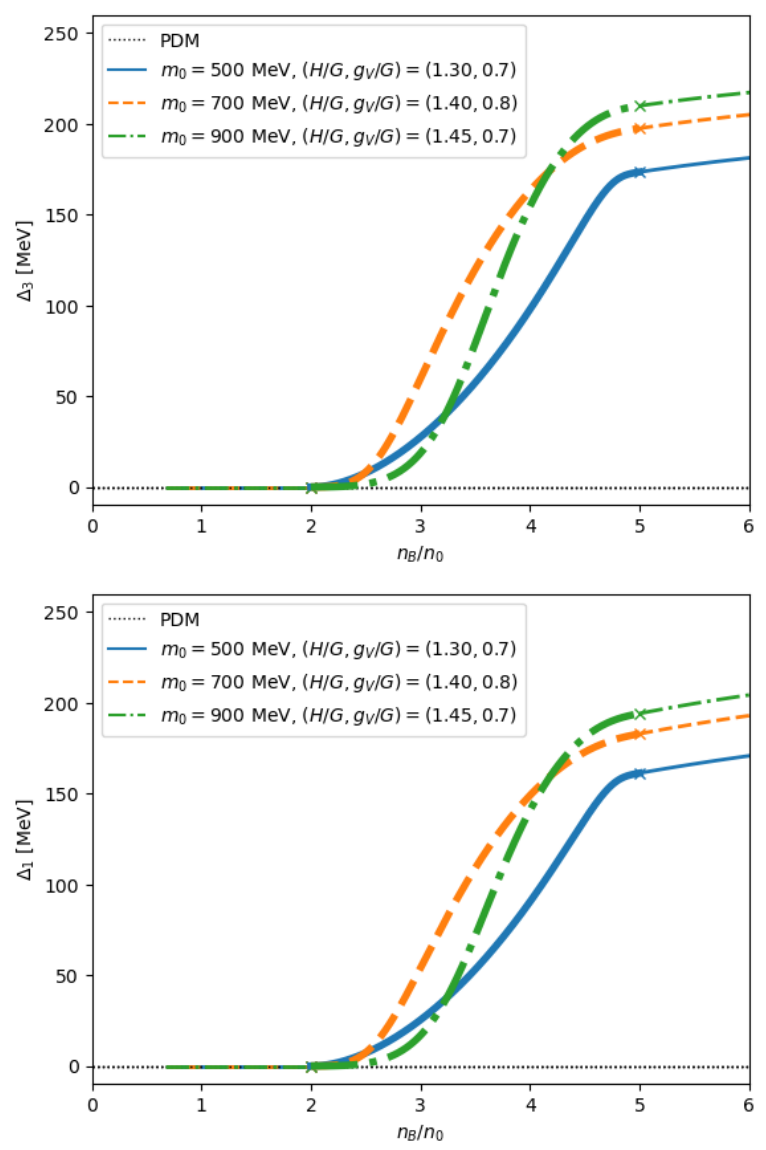

FIG. 6: Diquark condensates as functions of density; (upper) $\Delta_{3}=\Delta_{u d}$, and (lower) $\Delta_{1}=\Delta_{d s}$.

\section{Compositions}

Finally we examine the composition of matter including leptons. Figure8 shows the fraction of quark density for each flavor and lepton fraction per baryon density,

$$
\frac{n_{f}}{n_{B}}, \quad \frac{n_{l}}{n_{B}},
$$

where $f=u, d, s$, and the subscript $l$ refers to electron or muon densities. The lepton fraction is determined by the charge neutrality and $\beta$-equilibrium condition, which is controlled by the charge chemical potential $\mu_{Q}$.

One of distinct features in our unified model is the evolution of the strangeness fraction. The strangeness fraction becomes significant around $n_{B} \simeq 2.5 n_{0}$, and strange quarks are as abundant as up- and down-quarks at $n_{B} \gtrsim 4.5 n_{0}$. At $n_{B}=5 n_{0}$, the matter becomes the CFL quark matter where the sum of quark densities satisfies the charge neutrality condition; no leptons needed in the CFL phase. This trend with the strangeness differs from pure nuclear models in which lepton fraction increases as the baryon density does. 

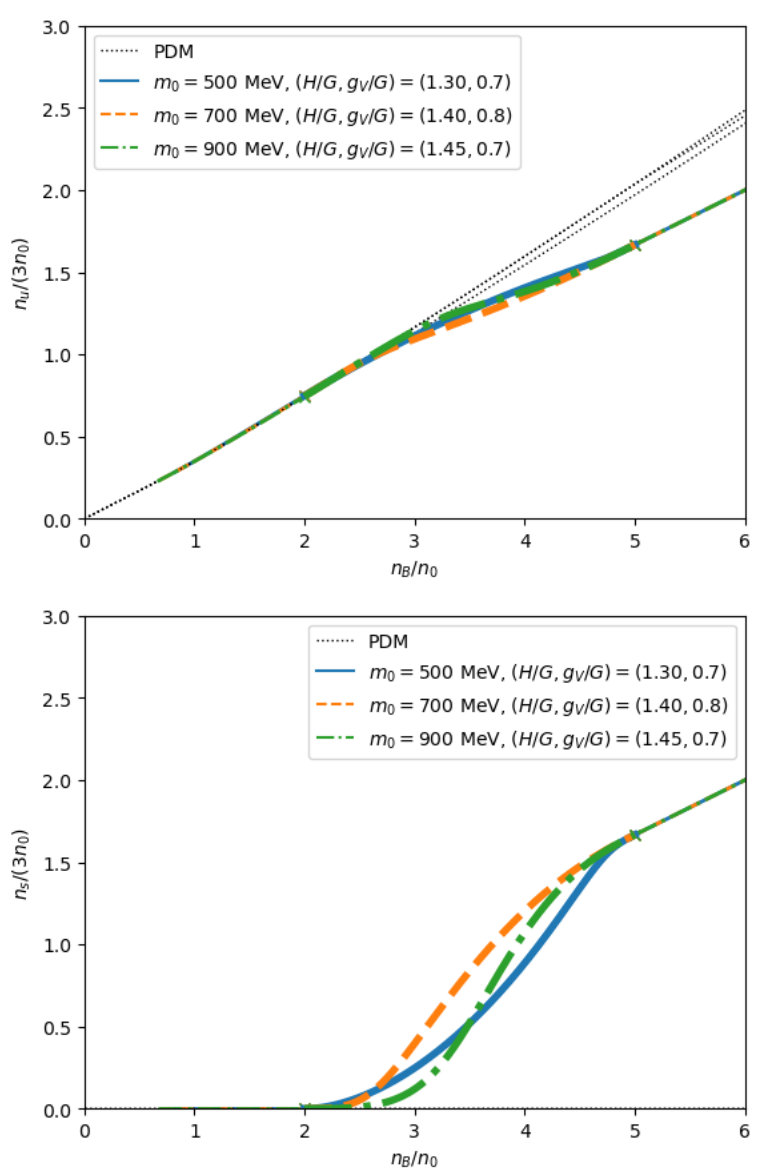

FIG. 7: Number density for (upper) up-quarks, and (lower) strange-quarks.

\section{DISCUSSIONS}

In this work, the chiral condensates in the crossover domain are based on the interpolation of those in nuclear and quark matter domains, so our pictures for the chiral restoration are somewhat indirect. Especially the role of confinement has been obscure. In this section we conjecture several scenarios to infer the microphysics in the interpolated domain.

\section{A. Casher's argument and the chiral scalar density in nucleons}

For the relation between confinement and chiral symmetry breaking, Casher suggested that the absence of the chiral symmetry breaking effects do not allow the descriptions of quark confinement [77. In our arguments we slightly relax Casher's arguments by just assuming the presence of chiral variant fields $(\sigma, \vec{\pi})$. The overall size of the chiral variant fields is characterized by $\phi^{2} \equiv\left(\sigma^{2}+\vec{\pi}^{2}\right)$ which is chiral invariant, while the direction of the four vector $(\sigma, \vec{\pi})$ is chiral variant.

The Casher's argument focus on the helicity for a mass-

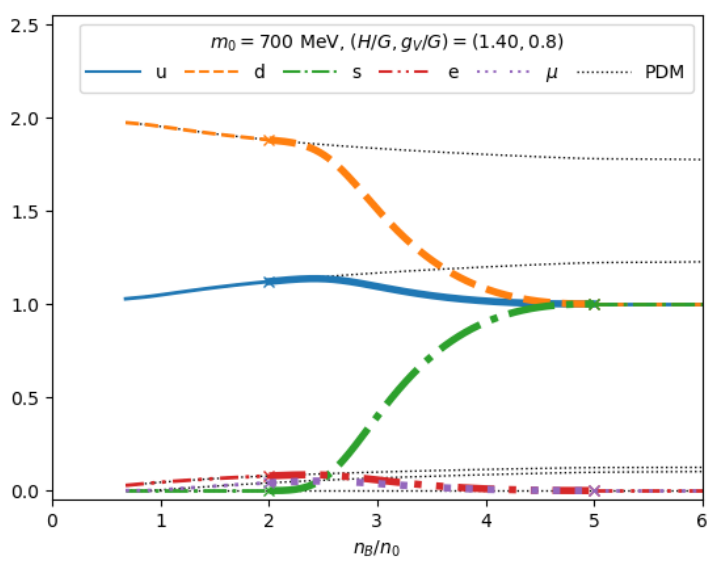

FIG. 8: Matter composition $n_{f} / n_{B}(f=u, d, s)$ and $n_{l} / n_{B}$ as functions of baryon density.

less quark. The confinement requires the massless quarks to change the directions, but it does not flip the spin, violating either the conservation of the helicity or angular momentum. To avoid such violation the confining boundaries must develop field ${ }^{3}$ which can carry the quanta of quarks just before the reflection, see Fig 9. The candidates of such fields are $\sigma$ and $\vec{\pi}$ which transform a left(right-)handed quark into a right-(left-)handed one, and at the same time the four vector $(\sigma, \vec{\pi})$ rotates to conserve the helicity. (This intuitive picture will be further developed in the next section.)

Now let us apply this picture and see what consequences would follow. Firstly the Casher's argument naturally leads to the chiral scalar density in hadrons. This may be related to the so-called in-hadron condensate [79. Meanwhile, to phrase the vacuum condensates in this context, one should first consider the $\sigma$ meson as a confined particle, and then argue the condensation of the $\sigma$ mesons. Once the space is filled with many $\sigma$, the quarks can flip the chirality anywhere.

\footnotetext{
${ }^{3}$ One can also think of the instantons as the sources of the chirality flipping; in this picture $\sigma$ appears due to quarks bound to instantons 78.
} 

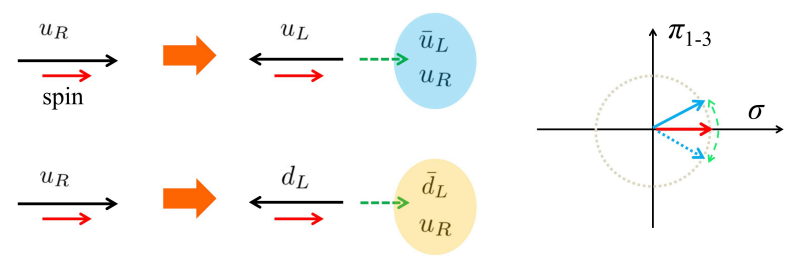

FIG. 9: Helicity and spin conservation due to the emergence of the $(\sigma, \vec{\pi})$ fields. The confinement turns a right-moving quark, $u_{R}$, into a left-moving quark, $u_{L}\left(d_{L}\right)$, but without changing the spin. The helicity is conserved by fields made of $\bar{u}_{L} u_{R}\left(\bar{d}_{L} u_{R}\right)$. The $(\sigma, \vec{\pi})$ fields fluctuate whenever the chirality of quarks flip.

\section{B. Topology of pion clouds and spatial modulations of the chiral scalar density}

The nucleon scalar density has the sign opposite to the vacuum chiral condensate but with the similar magnitude. Extrapolating this picture would lead to the expectation that, as nucleons overlap, they form a large domain with the positive chiral scalar density (negative $\sigma)$, dominating over the negative chiral scalar density (positive $\sigma$ ) from the vacuum, see Fig, 10. This consideration does not smoothly match with quark model descriptions at high density, where the chiral scalar density approaches zero, rather than the positive value. To understand this discrepancy, below we first consider a two baryon system and chiral scalar density in it.
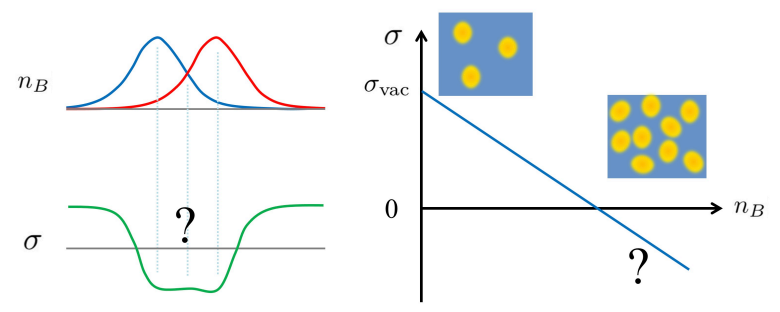

FIG. 10: Naive extrapolation of the linear density approximation for the chiral condensate. As baryons overlap, the positive chiral scalar density (negative $\sigma$ ) from baryons dominates over the negative scalar density from vacuum one $(\sigma>0)$.

For this purpose we first examine in more detail how the $\sigma$ field changes the sign from the vacuum to the inside of a nucleon. Considering that the effective Lagrangian is chiral symmetric, the effective potential is a function of $\phi^{2}$. It takes a minimum at $\phi=\sigma_{\mathrm{vac}}$ for the vacuum. We suppose that the field variation along this circle $(\phi=$ const.) does not cost much energy. Then the $\sigma$ around a nucleon should accompany $\pi$ fields whose magnitudes are
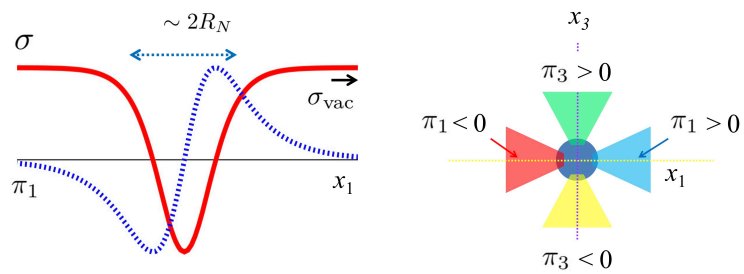

FIG. 11: The expected behavior of chiral scalar $(\sigma)$ and pseudo scalar fields $(\vec{\pi})$ near a nucleon (one dimensional slice). The chiral invariant combination of these fields are $\sigma^{2}+\vec{\pi}^{2} \simeq$ const. The right is the hedgehog profile.
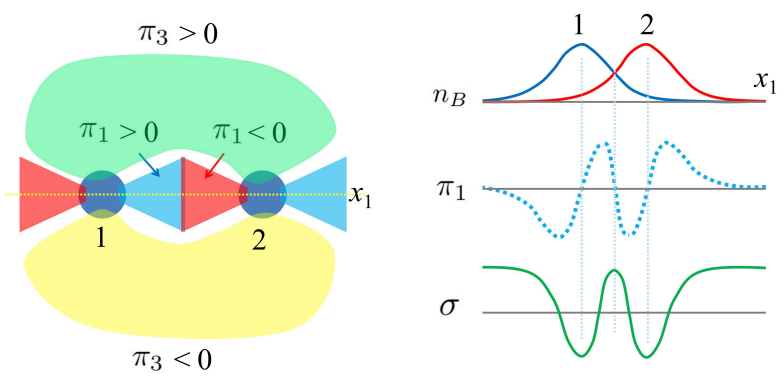

FIG. 12: Two nucleons at close distance, with the hedgehog pion configurations, where the vectors $\vec{\pi}$ and $\vec{x}$ are parallel. The rightmost part shows the baryon density, $\pi_{1}$ fields, and $\sigma$ fields along the $x_{1}$ axis. The negative $\sigma$ fields are accumulated at the center of baryons, while the positive $\sigma$ are accumulated at the interface of two baryons.

large near the surface of nucleons, and are vanishing at the center, as shown in Fig.11. The spatial average of $\pi$ fields is zero. If we arrange isospin distributions for these $\vec{\pi}$ fields properly to generate the topological number one as in the hedgehog form, we arrive at descriptions similar to the chiral soliton models 80 87.

The topological numbers of pions around nucleons give important constraints on how baryons come close together. Let us consider two nucleons which are close in distance (Fig 12). As we mentioned, in a naive description two domains of the positive scalar density simply merge to form one large domain with the positive scalar density (negative $\sigma$ ) as was shown in Fig.10. This picture is corrected by considering the topological constraint as shown in Fig.12. For two nucleon problems, the configuration of $(\sigma, \overrightarrow{\vec{\pi}})$ must have nodes to generate topological numbers tw 4 instead of zero. Hence $(\sigma, \vec{\pi})$ should have spatial modulations with positive and negative scalar densities, instead of forming single large domain with the positive scalar charge. As more and more

\footnotetext{
4 In (1+1)-dimensional models such statements become exact by applying the bosonization method 88 91; $n_{B} \sim \partial_{1} \varphi, \sigma \sim \cos \varphi$, and $\pi \sim \sin \varphi$ (for $U(1))$. Studies of dense systems can be found, e.g. in Refs. 92 . 95 .
} 
nucleons are packed, the spatial modulations of $(\sigma, \vec{\pi})$ fields become finer. The positive and negative $\sigma$ fields tend to cancel only in the spatial average. The magnitudes of the modulations are controlled by the size of $\phi$, not by the value of $\sigma$. As far as $\phi$ is nonzero, it is natural to expect that the nucleon and constituent quark masses are substantial, but still we can describe the chiral restoration in the sense of $\int_{\mathbf{x}} \sigma(x)=0$. This picture fits to the concept of the chiral invariant mass in the PDM which can be nonzero even for $\sigma=0$ as discussed in Refs. 96, 97.

In the above argument we address the possibility that each nucleon accompanies the spatial modulations in $\sigma$ and $\vec{\pi}$ in a topological configuration. (For chiral density waves in the PDM, see Refs. 35, 45, 46. ) In compressing nuclear matter, such modulations do not cancel, but just get squeezed altogether as far as $\phi$ remains finite. This picture shares several viewpoints with the scenarios of the soliton crystals [98,102] or chiral spirals [76, 103 112 ; if the compressed nucleons favor the periodic structure, they also lead to the lattice in $(\sigma, \vec{\pi})$. Such inhomogeneous chiral condensates have been discussed in both nucleonic models and quark models. Whether they can connect by the quark-hadron continuity is an interesting question, see Ref. [113] and references therein (Refs. cited) for instance.

\section{Diquarks}

Finally we consider how diquark condensates develop from nuclear to quark matter. As we have assumed that nucleon fields (before the diagonalization of the mass matrix) transform as $(1 / 2,0)_{L} \oplus(0,1 / 2)_{R}$ in terms of the chiral multiplet, a simple way to combine three quarks is to first consider diquarks in the color antitriplet representation and then put a leftover quark. The diquarks

$$
\left[u_{L} d_{L}\right]_{I=0}, \quad\left[u_{R} d_{R}\right]_{I=0},
$$

are $S U(2)_{L}$ and $S U(2)_{R}$ singlet, respectively, and hence are invariant under the chiral transformations for $S U(2)_{L} \otimes S U(2)_{R}$. We attach the leftover quark to these diquarks, then the resulting nucleon follows the chiral transformation of the leftover quark. Below we further assume that the diquark takes $J^{P}=0^{+}$and the spatial S-wave, for which the attractive correlations are the largest. In this respect, the nucleon already contains the source of diquark condensates for the high density matter.

At low density the diquark correlations may exist but a diquark is tightly bound to a leftover quark to form a nucleon. In this regime the diquarks are hidden inside of nucleons; the diquark fields may be associated with nucleons, but they do not take the form of condensates which spread over the system. The bulk properties of the system are described in terms of nucleons. The trend begins to change when quark exchanges between nucleons become very frequent. Here diquarks are available not only as constituents of nucleons, but as participants to the bulk properties of the system. As the diquark fields overlap, they develop the coherence; the phase of condensates, $\varphi$ in $\Delta=|\Delta| \mathrm{e}^{i \varphi}$, correlate over long distance. In these pictures, the diquark condensates begin to develop when nuclear many-body forces become important $\left(n_{B} \gtrsim 2 n_{0}\right)$, gradually develop toward quark matter, and get established at high density $\left(n_{B} \gtrsim 5 n_{0}\right)^{5}$.

Based on the same picture, we expect that diquark fields with strangeness are introduced to the system as the constituents of hyperons. Then the diquark fields gradually develop as the hyperons dissociate through the quark exchanges.

\section{SUMMARY}

In this paper, we have elaborated a recipe to compute various condensates in the domain between nuclear and quark matter, assuming the quark-hadron continuity picture. After looking at the behaviors of these condensates, we then conjecture qualitative scenarios which interpolate nuclear and quark matter descriptions.

We found that the PDM with the substantial chiral invariant mass, $m_{0} \gtrsim 500 \mathrm{MeV}$, has a number of favorable properties in descriptions for EOS (as found in the previous works) and also for various condensates. In particular the substantial reduction of $\sigma$ in dilute regime (driven by the positive scalar charge in nucleons) does not change the nucleon properties drastically. This is consistent with our neglect of the nucleon Dirac sea and use of fixed nucleon-meson couplings for $n_{B} \lesssim 2 n_{0}$. In our view, the intrinsic properties of nucleons begin to substantially change at $n_{B} \gtrsim 2 n_{0}$, where quark exchanges among baryons become frequent; since baryons are made of quarks, the quark exchanges are supposed to change the baryon structure. The quarks, which are partially released, are also affected by the medium and should change the properties such as the effective mass. The direct descriptions of such changes is difficult, but at least we can constrain it through the quark matter constraints at high density. Our interpolation scheme is a practical way to implement these ideas.

This work has addressed only few aspects on the chiral symmetry in dense matter. There remain many issues to be addressed. Here we list up some:

(i) The PDM model can be extended to include hyperons 33, 117 121. For NS matter, the charge chemical potential $\mu_{Q}$ ranges from $-100 \mathrm{MeV}$ to $-200 \mathrm{MeV}$

\footnotetext{
5 The onset of diquark pair condensations may be constrained by NS cooling. In particular, some cooling curves disfavor substantial CFL cores which would induce too rapid cooling [114, 115. One possible interpretation is that such observed NSs are too light and do not have large core densities. But if those NSs turn out to be heavy and have large core densities, they put stringent constraints on the CFL phase. For cooling of accreting NSs, see Ref. 116].
} 
around $n_{B} \sim 1-2 n_{0}$, and hyperons may appear for $2-3 n_{0}$, not far from our choice for the hadronic boundary $n_{B}=2 n_{0}$ (see for instance Sec.III in Ref. 122]). The slight extension from $n_{B}=2 n_{0}$ to higher density and manifest treatment of hyperons would give more concrete descriptions of the strangeness than in this work.

(ii) The detailed understanding of a nucleon and its meson cloud should give a guide on the chiral symmetry in dense matter. An important question in the context of the PDM is how a $(\sigma, \vec{\pi})$ cloud differs for the positive $(N(939))$ and negative parity $(N(1535))$ nucleons. In terms of the constituent quark models, $N(1535)$ contains the $\mathrm{P}$-wave excitation of a quark, with the larger spatial size than $N(939)$. How this size scale estimate and $\sigma$ in the PDM are related is an important question to understand the medium effects in the PDM. In general the medium effects should influence hadrons with the larger size, as they have closer in distance to the other hadrons.

(iii) Ultimately our patchwork of nuclear and quark matter descriptions should be replaced with a description based on a single model. Baryons should be constructed explicitly in terms of quarks. Several recent works [123126], although schematic, have given concrete descriptions of quarks from nuclear to quark matter domains. One of the important outcome is the peak in the speed of sound [124 126] which has been a puzzling feature inferred from NS observations [127. But detailed questions such as the fate of chiral symmetry breaking or diquark correlations have not been addressed in such modelings. Our descriptions in this paper should give some hints for the fuller understanding of nuclear-quark matter phase transitions.

\section{ACKNOWLEDGMENTS}

The work of T.M. and M.H. was supported in part by JSPS KAKENHI Grant No. 20K03927. T.M. was also supported in part by the Department of Physics, Nagoya University. T.K. was supported by NSFC Grant No. 11875144.

\section{Appendix A: Calculation of $\partial a_{n} / \partial J$ in Eq.42}

We write $\vec{P}$ as the vector which has the components of the six values of $\partial^{k} P /\left(\partial \mu_{B}\right)^{k}(k=0,1,2)$ at the boundaries $\mu_{B}=\mu_{B}^{L}, \mu_{B}^{U}$ calculated from the PDM and the NJL model. Since $P_{I}$ is a polynomial of $\mu_{B}$, the vector of the values of $\partial^{k} P_{\mathrm{I}} /\left(\partial \mu_{B}\right)^{k}(k=0,1,2)$ at the boundaries is represented as $M \vec{a}$, where $\vec{a}=\left(a_{n}\right)$ and $M$ is a matrix of $\mu_{B}^{L}, \mu_{B}^{U}$. Therefore, the $J$ derivative of $a_{n}$ is calculated as

$$
\frac{\partial a_{n}}{\partial J}=-M^{-1} \frac{\partial M}{\partial J} M^{-1} \vec{P}+M^{-1} \frac{\partial \vec{P}}{\partial J} .
$$

Since $\vec{P}$ is evaluated at the boundaries, $\partial \vec{P} / \partial J$ is calculated as, for example,

$$
\begin{aligned}
\frac{\partial}{\partial J}\left(\left.P\right|_{\mu_{B}^{L}}\right) & =\left.\frac{\partial \mu_{B}^{L}}{\partial J} \frac{\partial P}{\partial \mu_{B}}\right|_{\mu_{B}^{L}}+\left.\frac{\partial P}{\partial J}\right|_{\mu_{B}^{L}}, \\
\frac{\partial}{\partial J}\left(\left.\frac{\partial P}{\partial \mu_{B}}\right|_{\mu_{B}^{L}}\right) & =\left.\frac{\partial \mu_{B}^{L}}{\partial J} \frac{\partial^{2} P}{\partial \mu_{B}^{2}}\right|_{\mu_{B}^{L}}+\left.\frac{\partial^{2} P}{\partial J \partial \mu_{B}}\right|_{\mu_{B}^{L}}, \\
\frac{\partial}{\partial J}\left(\left.\frac{\partial^{2} P}{\partial \mu_{B}^{2}}\right|_{\mu_{B}^{L}}\right) & =\left.\frac{\partial \mu_{B}^{L}}{\partial J} \frac{\partial^{3} P}{\partial \mu_{B}^{3}}\right|_{\mu_{B}^{L}}+\left.\frac{\partial^{3} P}{\partial J \partial \mu_{B}^{2}}\right|_{\mu_{B}^{L}} .
\end{aligned}
$$

Since the density at the boundaries are fixed for any $J$, Eq. A3 equals zero and

$$
\frac{\partial \mu_{B L}}{\partial J}=-\left.\frac{\partial^{2} P}{\partial J \partial \mu_{B}}\right|_{\mu_{B}^{L}} /\left.\frac{\partial^{2} P}{\partial \mu_{B}^{2}}\right|_{\mu_{B}^{L}} .
$$

Moreover, $\partial^{k+1} P / \partial J\left(\partial \mu_{B}\right)^{k}=-\partial^{k} \phi /\left(\partial \mu_{B}\right)^{k}$ is determined from the derivation of the gap equation, for example,

$$
0=\frac{\partial}{\partial J}\left(\left.\frac{\partial \Omega}{\partial \phi}\right|_{\phi_{*}}\right)=\left.\frac{\partial \phi_{*}}{\partial J} \frac{\partial^{2} \Omega}{\partial \phi^{2}}\right|_{\phi_{*}}+\left.\frac{\partial^{2} \Omega}{\partial J \partial \phi}\right|_{\phi_{*}}
$$

where $\phi_{*}$ is the solution of the gap equation.
[1] Y. Aoki, S. Borsanyi, S. Durr, Z. Fodor, S. D. Katz, S. Krieg, and K. K. Szabo, The QCD transition temperature: results with physical masses in the continuum limit II., JHEP 06, 088, arXiv:0903.4155 [hep-lat].

[2] C. Bonati, M. D'Elia, M. Mariti, M. Mesiti, F. Negro, and F. Sanfilippo, Curvature of the chiral pseudocritical line in QCD: Continuum extrapolated results, Phys. Rev. D 92, 054503 (2015), arXiv:1507.03571 [hep-lat].

[3] A. Bazavov et al. (HotQCD), Chiral crossover in QCD at zero and non-zero chemical potentials, Phys. Lett. B 795, 15 (2019), arXiv:1812.08235 [hep-lat]

[4] G. Baym, T. Hatsuda, T. Kojo, P. D. Powell, Y. Song, and T. Takatsuka, From hadrons to quarks in neutron stars: a review, Rept. Prog. Phys. 81, 056902 (2018)
arXiv:1707.04966 [astro-ph.HE]

[5] T. Kojo, QCD equations of state and speed of sound in neutron stars, AAPPS Bull. 31, 11 (2021), arXiv:2011.10940 [nucl-th]

[6] Z. Arzoumanian et al. (NANOGrav), The NANOGrav 11-year Data Set: High-precision timing of 45 Millisecond Pulsars, Astrophys. J. Suppl. 235, 37 (2018), arXiv:1801.01837 [astro-ph.HE],

[7] E. Fonseca et al., The NANOGrav Nine-year Data Set: Mass and Geometric Measurements of Binary Millisecond Pulsars, Astrophys. J. 832, 167 (2016), arXiv:1603.00545 [astro-ph.HE].

[8] P. Demorest, T. Pennucci, S. Ransom, M. Roberts, and J. Hessels, Shapiro Delay Measurement of A Two 
Solar Mass Neutron Star, Nature 467, 1081 (2010) arXiv:1010.5788 [astro-ph.HE].

[9] J. Antoniadis et al., A Massive Pulsar in a Compact Relativistic Binary, Science 340, 6131 (2013) arXiv:1304.6875 [astro-ph.HE].

[10] E. Fonseca et al., Refined Mass and Geometric Measurements of the High-mass PSR J0740+6620, Astrophys. J. Lett. 915, L12 (2021), arXiv:2104.00880 [astro-ph.HE]

[11] B. Abbott et al. (LIGO Scientific, Virgo), GW170817: Observation of Gravitational Waves from a Binary Neutron Star Inspiral, Phys. Rev. Lett. 119, 161101 (2017), arXiv:1710.05832 [gr-qc]

[12] M. C. Miller et al., The Radius of PSR J0740+6620 from NICER and XMM-Newton Data, Astrophys. J. Lett. 918, L28 (2021), arXiv:2105.06979 [astro-ph.HE].

[13] T. E. Riley et al., A NICER View of the Massive Pulsar PSR J0740+6620 Informed by Radio Timing and XMM-Newton Spectroscopy, Astrophys. J. Lett. 918, L27 (2021), arXiv:2105.06980 [astro-ph.HE]

[14] G. Raaijmakers, S. K. Greif, K. Hebeler, T. Hinderer, S. Nissanke, A. Schwenk, T. E. Riley, A. L. Watts, J. M. Lattimer, and W. C. G. Ho, Constraints on the Dense Matter Equation of State and Neutron Star Properties from NICER's Mass-Radius Estimate of PSR J0740+6620 and Multimessenger Observations, Astrophys. J. Lett. 918, L29 (2021), arXiv:2105.06981 [astroph.HE]

[15] K. Masuda, T. Hatsuda, and T. Takatsuka, Hadron-Quark Crossover and Massive Hybrid Stars with Strangeness, Astrophys. J. 764, 12 (2013), arXiv:1205.3621 [nucl-th]

[16] K. Masuda, T. Hatsuda, and T. Takatsuka, Hadronquark crossover and massive hybrid stars, PTEP 2013, 073D01 (2013), arXiv:1212.6803 [nucl-th]

[17] K. Masuda, T. Hatsuda, and T. Takatsuka, Hyperon Puzzle, Hadron-Quark Crossover and Massive Neutron Stars, Eur. Phys. J. A 52, 65 (2016), arXiv:1508.04861 [nucl-th]

[18] T. Kojo, P. D. Powell, Y. Song, and G. Baym, Phenomenological QCD equation of state for massive neutron stars, Phys. Rev. D 91, 045003 (2015), arXiv:1412.1108 [hep-ph]

[19] K. Fukushima and T. Kojo, The Quarkyonic Star, Astrophys. J. 817, 180 (2016) arXiv:1509.00356 [nucl-th]

[20] G. Baym, S. Furusawa, T. Hatsuda, T. Kojo, and H. Togashi, New Neutron Star Equation of State with Quark-Hadron Crossover, Astrophys. J. 885, 42 (2019), arXiv:1903.08963 [astro-ph.HE].

[21] T. Minamikawa, T. Kojo, and M. Harada, Quarkhadron crossover equations of state for neutron stars: constraining the chiral invariant mass in a parity doublet model, Phys. Rev. C 103, 045205 (2021). arXiv:2011.13684 [nucl-th],

[22] C. E. Detar and T. Kunihiro, Linear $\sigma$ Model With Parity Doubling, Phys. Rev. D 39, 2805 (1989).

[23] D. Jido, M. Oka, and A. Hosaka, Chiral symmetry of baryons, Prog. Theor. Phys. 106, 873 (2001), arXiv:hep$\mathrm{ph} / 0110005$

[24] T. Hatsuda and M. Prakash, Parity Doubling of the Nucleon and First Order Chiral Transition in Dense Matter, Phys. Lett. B 224, 11 (1989)

[25] D. Zschiesche, L. Tolos, J. Schaffner-Bielich, and R. D. Pisarski, Cold, dense nuclear matter in a $\mathrm{SU}(2)$ parity doublet model, Phys. Rev. C 75, 055202 (2007),
arXiv:nucl-th/0608044

[26] V. Dexheimer, S. Schramm, and D. Zschiesche, Nuclear matter and neutron stars in a parity doublet model, Phys. Rev. C 77, 025803 (2008), arXiv:0710.4192 [nuclth]

[27] V. Dexheimer, G. Pagliara, L. Tolos, J. SchaffnerBielich, and S. Schramm, Neutron stars within the SU(2) parity doublet model, Eur. Phys. J. A 38, 105 (2008), arXiv:0805.3301 [nucl-th]

[28] C. Sasaki and I. Mishustin, Thermodynamics of dense hadronic matter in a parity doublet model, Phys. Rev. C 82, 035204 (2010), arXiv:1005.4811 [hep-ph].

[29] C. Sasaki, H. K. Lee, W.-G. Paeng, and M. Rho, Conformal anomaly and the vector coupling in dense matter, Phys. Rev. D 84, 034011 (2011), arXiv:1103.0184 [hep-ph].

[30] S. Gallas, F. Giacosa, and G. Pagliara, Nuclear matter within a dilatation-invariant parity doublet model: the role of the tetraquark at nonzero density, Nucl. Phys. A 872, 13 (2011), arXiv:1105.5003 [hep-ph].

[31] W.-G. Paeng, H. K. Lee, M. Rho, and C. Sasaki, Dilaton-Limit Fixed Point in Hidden Local Symmetric Parity Doublet Model, Phys. Rev. D 85, 054022 (2012), arXiv:1109.5431 [hep-ph].

[32] J. Steinheimer, S. Schramm, and H. Stocker, The hadronic SU(3) Parity Doublet Model for Dense Matter, its extension to quarks and the strange equation of state, Phys. Rev. C 84, 045208 (2011), arXiv:1108.2596 [hep-ph]

[33] V. Dexheimer, J. Steinheimer, R. Negreiros, and S. Schramm, Hybrid Stars in an SU(3) parity doublet model, Phys. Rev. C 87, 015804 (2013), arXiv:1206.3086 [astro-ph.HE]

[34] W.-G. Paeng, H. K. Lee, M. Rho, and C. Sasaki, Interplay between $\omega$-nucleon interaction and nucleon mass in dense baryonic matter, Phys. Rev. D 88, 105019 (2013), arXiv:1303.2898 [nucl-th]

[35] A. Heinz, F. Giacosa, and D. H. Rischke, Chiral density wave in nuclear matter, Nucl. Phys. A 933, 34 (2015), arXiv:1312.3244 [nucl-th]

[36] Y. Motohiro, Y. Kim, and M. Harada, Asymmetric nuclear matter in a parity doublet model with hidden local symmetry, Phys. Rev. C 92, 025201 (2015), [Erratum: Phys.Rev.C 95, 059903 (2017)], arXiv:1505.00988 [nuclth]

[37] S. Benic, I. Mishustin, and C. Sasaki, Effective model for the QCD phase transitions at finite baryon density, Phys. Rev. D 91, 125034 (2015), arXiv:1502.05969 [hep$\mathrm{ph}]$.

[38] A. Mukherjee, J. Steinheimer, and S. Schramm, Higherorder baryon number susceptibilities: interplay between the chiral and the nuclear liquid-gas transitions, Phys. Rev. C 96, 025205 (2017), arXiv:1611.10144 [nucl-th].

[39] A. Mukherjee, S. Schramm, J. Steinheimer, and V. Dexheimer, The application of the Quark-Hadron Chiral Parity-Doublet Model to neutron star matter, Astron. Astrophys. 608, A110 (2017), arXiv:1706.09191 [nuclth]

[40] D. Suenaga, Examination of $N^{*}(1535)$ as a probe to observe the partial restoration of chiral symmetry in nuclear matter, Phys. Rev. C 97, 045203 (2018), arXiv:1704.03630 [nucl-th]

[41] Y. Takeda, Y. Kim, and M. Harada, Catalysis of partial chiral symmetry restoration by $\Delta$ matter, Phys. Rev. C 
97, 065202 (2018), arXiv:1704.04357 [nucl-th]

[42] M. Marczenko and C. Sasaki, Net-baryon number fluctuations in the Hybrid Quark-Meson-Nucleon model at finite density, Phys. Rev. D 97, 036011 (2018), arXiv:1711.05521 [hep-ph]

[43] W.-G. Paeng, T. T. S. Kuo, H. K. Lee, Y.-L. Ma, and M. Rho, Scale-invariant hidden local symmetry, topology change, and dense baryonic matter. II., Phys. Rev. D 96, 014031 (2017), arXiv:1704.02775 [nucl-th].

[44] M. Marczenko, D. Blaschke, K. Redlich, and C. Sasaki, Chiral symmetry restoration by parity doubling and the structure of neutron stars, Phys. Rev. D 98, 103021 (2018), arXiv:1805.06886 [nucl-th].

[45] H. Abuki, Y. Takeda, and M. Harada, Dual chiral density waves in nuclear matter, EPJ Web Conf. 192, 00020 (2018), arXiv:1809.06485 [hep-ph].

[46] Y. Takeda, H. Abuki, and M. Harada, Novel dual chiral density wave in nuclear matter based on a parity doublet structure, Phys. Rev. D 97, 094032 (2018), arXiv:1803.06779 [hep-ph]

[47] T. Yamazaki and M. Harada, Constraint to chiral invariant masses of nucleons from GW170817 in an extended parity doublet model, Phys. Rev. C 100, 025205 (2019), arXiv:1901.02167 [nucl-th].

[48] M. Harada and T. Yamazaki, Charmed Mesons in Nuclear Matter Based on Chiral Effective Models, JPS Conf. Proc. 26, 024001 (2019)

[49] M. Marczenko, D. Blaschke, K. Redlich, and C. Sasaki, Parity Doubling and the Dense Matter Phase Diagram under Constraints from Multi-Messenger Astronomy, Universe 5, 180 (2019), arXiv:1905.04974 [nucl-th].

[50] M. Harada, Dense nuclear matter based on a chiral model with parity doublet structure, in 18th International Conference on Hadron Spectroscopy an (2020).

[51] M. Marczenko, D. Blaschke, K. Redlich, and C. Sasaki, Toward a unified equation of state for multi-messenger astronomy, Astron. Astrophys. 643, A82 (2020), arXiv:2004.09566 [astro-ph.HE].

[52] G. Aarts, C. Allton, D. De Boni, S. Hands, B. Jäger, C. Praki, and J.-I. Skullerud, Light baryons below and above the deconfinement transition: medium effects and parity doubling, JHEP 06, 034, arXiv:1703.09246 [heplat]

[53] J. D. Walecka, A Theory of highly condensed matter, Annals Phys. 83, 491 (1974)

[54] B. D. Serot and J. D. Walecka, The Relativistic Nuclear Many Body Problem, Adv. Nucl. Phys. 16, 1 (1986).

[55] B. D. Serot and J. D. Walecka, Recent progress in quantum hadrodynamics, Int. J. Mod. Phys. E 6, 515 (1997), arXiv:nucl-th/9701058.

[56] T. Hatsuda and T. Kunihiro, QCD phenomenology based on a chiral effective Lagrangian, Phys. Rept. 247, 221 (1994), arXiv:hep-ph/9401310

[57] M. Kitazawa, T. Koide, T. Kunihiro, and Y. Nemoto, Chiral and color superconducting phase transitions with vector interaction in a simple model, Prog. Theor. Phys. 108, 929 (2002), [Erratum: Prog.Theor.Phys. 110, 185186 (2003)], arXiv:hep-ph/0207255.

[58] N. M. Bratovic, T. Hatsuda, and W. Weise, Role of Vector Interaction and Axial Anomaly in the PNJL Modeling of the QCD Phase Diagram, Phys. Lett. B 719, 131 (2013), arXiv:1204.3788 [hep-ph].
[59] M. G. Alford, A. Schmitt, K. Rajagopal, and T. Schäfer, Color superconductivity in dense quark matter, Rev. Mod. Phys. 80, 1455 (2008), arXiv:0709.4635 [hep-ph]

[60] P. Gubler and D. Satow, Recent Progress in QCD Condensate Evaluations and Sum Rules, Prog. Part. Nucl. Phys. 106, 1 (2019) arXiv:1812.00385 [hep-ph]

[61] J. Gasser, H. Leutwyler, and M. E. Sainio, Sigma term update, Phys. Lett. B 253, 252 (1991).

[62] P. Schweitzer, The Sigma term form-factor of the nucleon in the large N(C) limit, Phys. Rev. D 69, 034003 (2004), arXiv:hep-ph/0307336.

[63] N. Kaiser, P. de Homont, and W. Weise, In-medium chiral condensate beyond linear density approximation, Phys. Rev. C 77, 025204 (2008), arXiv:0711.3154 [nuclth]

[64] N. Kaiser and W. Weise, Chiral condensate in neutron matter, Phys. Lett. B 671, 25 (2009), arXiv:0808.0856 [nucl-th].

[65] M. Drews and W. Weise, Functional renormalization group studies of nuclear and neutron matter, Prog. Part. Nucl. Phys. 93, 69 (2017), arXiv:1610.07568 [nucl-th],

[66] F. Karsch, K. Redlich, and A. Tawfik, Thermodynamics at nonzero baryon number density: A Comparison of lattice and hadron resonance gas model calculations, Phys. Lett. B 571, 67 (2003), arXiv:hep-ph/0306208.

[67] A. Andronic, P. Braun-Munzinger, K. Redlich, and J. Stachel, Decoding the phase structure of QCD via particle production at high energy, Nature 561, 321 (2018), arXiv:1710.09425 [nucl-th].

[68] E. V. Shuryak, Two Scales and Phase Transitions in Quantum Chromodynamics, Phys. Lett. B 107, 103 (1981).

[69] A. Manohar and H. Georgi, Chiral Quarks and the Nond Struateląivistic Quark Model, Nucl. Phys. B 234, 189 (1984).

[70] D. Suenaga and T. Kojo, Gluon propagator in two-color dense QCD: Massive Yang-Mills approach at one-loop, Phys. Rev. D 100, 076017 (2019), arXiv:1905.08751 [hep-ph].

[71] A. De Rujula, H. Georgi, and S. L. Glashow, Hadron Masses in a Gauge Theory, Phys. Rev. D 12, 147 (1975).

[72] A. Park, W. Park, and S. H. Lee, Tribaryon configurations and the inevitable three nucleon repulsions at short distance, Phys. Rev. D 98, 034001 (2018), arXiv:1801.10350 [hep-ph].

[73] A. Park, S. H. Lee, T. Inoue, and T. Hatsuda, Baryon-baryon interactions at short distances: constituent quark model meets lattice QCD, Eur. Phys. J. A 56, 93 (2020), arXiv:1907.06351 [hep-ph].

[74] A. Ayriyan, D. Blaschke, A. G. Grunfeld, D. AlvarezCastillo, H. Grigorian, and V. Abgaryan, Bayesian analysis of multimessenger $\mathrm{M}-\mathrm{R}$ data with interpolated hybrid EoS, Eur. Phys. J. A 57, 318 (2021), arXiv:2102.13485 [astro-ph.HE].

[75] Y. Song, G. Baym, T. Hatsuda, and T. Kojo, Effective repulsion in dense quark matter from nonperturbative gluon exchange, Phys. Rev. D 100, 034018 (2019), arXiv:1905.01005 [astro-ph.HE].

[76] T. Kojo, Y. Hidaka, L. McLerran, and R. D. Pisarski, Quarkyonic Chiral Spirals, Nucl. Phys. A 843, 37 (2010), arXiv:0912.3800 [hep-ph].

[77] A. Casher, Chiral Symmetry Breaking in Quark Confining Theories, Phys. Lett. B 83, 395 (1979).

[78] T. Schäfer and E. V. Shuryak, Instantons in QCD, Rev. Mod. Phys. 70, 323 (1998), arXiv:hep-ph/9610451. 
[79] S. J. Brodsky, C. D. Roberts, R. Shrock, and P. C. Tandy, Essence of the vacuum quark condensate, Phys. Rev. C 82, 022201 (2010), arXiv:1005.4610 [nucl-th].

[80] T. H. R. Skyrme, A Nonlinear field theory, Proc. Roy. Soc. Lond. A 260, 127 (1961).

[81] G. S. Adkins, C. R. Nappi, and E. Witten, Static Properties of Nucleons in the Skyrme Model, Nucl. Phys. B 228, 552 (1983)

[82] D. Diakonov, V. Y. Petrov, and P. V. Pobylitsa, A Chiral Theory of Nucleons, Nucl. Phys. B 306, 809 (1988)

[83] A. V. Manohar, Equivalence of the Chiral Soliton and Quark Models in Large N, Nucl. Phys. B 248, 19 (1984).

[84] S. Kahana, G. Ripka, and V. Soni, Soliton with Valence Quarks in the Chiral Invariant Sigma Model, Nucl. Phys. A 415, 351 (1984).

[85] I. Zahed and G. E. Brown, The Skyrme Model, Phys. Rept. 142, 1 (1986)

[86] H. Hata, T. Sakai, S. Sugimoto, and S. Yamato, Baryons from instantons in holographic QCD, Prog. Theor. Phys. 117, 1157 (2007), arXiv:hep-th/0701280.

[87] K. Nawa, H. Suganuma, and T. Kojo, Baryons in holographic QCD, Phys. Rev. D 75, 086003 (2007), arXiv:hep-th/0612187.

[88] E. Witten, Nonabelian Bosonization in TwoDimensions, Commun. Math. Phys. 92, 455 (1984).

[89] E. Witten, Chiral Symmetry, the 1/n Expansion, and the SU(N) Thirring Model, Nucl. Phys. B 145, 110 (1978)

[90] I. Affleck, On the Realization of Chiral Symmetry in (1+1)-dimensions, Nucl. Phys. B 265, 448 (1986).

[91] I. Affleck, Exact Critical Exponents for Quantum Spin Chains, Nonlinear Sigma Models at Theta $=$ pi and the Quantum Hall Effect, Nucl. Phys. B 265, 409 (1986)

[92] V. Schon and M. Thies, Emergence of Skyrme crystal in Gross-Neveu and 't Hooft models at finite density, Phys. Rev. D 62, 096002 (2000), arXiv:hep-th/0003195

[93] B. Bringoltz, Chiral crystals in strong-coupling lattice QCD at nonzero chemical potential, JHEP 03, 016 arXiv:hep-lat/0612010.

[94] T. Kojo, Chiral Spirals from Noncontinuous Chiral Symmetry: The Gross-Neveu model results, Phys. Rev. D 90, 065030 (2014), arXiv:1406.4630 [hep-ph].

[95] T. Kojo, A (1+1) dimensional example of Quarkyonic matter, Nucl. Phys. A 877, 70 (2012), arXiv:1106.2187 [hep-ph].

[96] Y.-L. Ma, M. Harada, H. K. Lee, Y. Oh, B.-Y. Park, and M. Rho, Dense baryonic matter in the hidden local symmetry approach: Half-skyrmions and nucleon mass, Phys. Rev. D 88, 014016 (2013), [Erratum: Phys.Rev.D 88, 079904 (2013)], arXiv:1304.5638 [hep-ph].

[97] M. Harada, H. K. Lee, Y.-L. Ma, and M. Rho, Inhomogeneous quark condensate in compressed Skyrmion matter, Phys. Rev. D 91, 096011 (2015), arXiv:1502.02508 [hep-ph]

[98] I. R. Klebanov, Nuclear Matter in the Skyrme Model, Nucl. Phys. B 262, 133 (1985)

[99] M. Rho, S.-J. Sin, and I. Zahed, Dense QCD: A Holographic Dyonic Salt, Phys. Lett. B 689, 23 (2010), arXiv:0910.3774 [hep-th].

[100] K.-Y. Kim, S.-J. Sin, and I. Zahed, Dense holographic QCD in the Wigner-Seitz approximation, JHEP 09, 001, arXiv:0712.1582 [hep-th].

[101] H. Forkel, A. D. Jackson, M. Rho, C. Weiss, A. Wirzba, and H. Bang, Chiral Symmetry Restoration and the
Skyrme Model, Nucl. Phys. A 504, 818 (1989).

[102] K. Nawa, H. Suganuma, and T. Kojo, Brane-induced Skyrmion on $\mathrm{S}^{* *} 3$ : Baryonic matter in holographic QCD, Phys. Rev. D 79, 026005 (2009), arXiv:0810.1005 [hep-th].

[103] M. Buballa and S. Carignano, Inhomogeneous chiral condensates, Prog. Part. Nucl. Phys. 81, 39 (2015), arXiv:1406.1367 [hep-ph].

[104] D. V. Deryagin, D. Y. Grigoriev, and V. A. Rubakov, Standing wave ground state in high density, zero temperature QCD at large N(c), Int. J. Mod. Phys. A 7, 659 (1992).

[105] D. Nickel, Inhomogeneous phases in the Nambu-JonaLasino and quark-meson model, Phys. Rev. D 80, 074025 (2009), arXiv:0906.5295 [hep-ph].

[106] S. Carignano, D. Nickel, and M. Buballa, Influence of vector interaction and Polyakov loop dynamics on inhomogeneous chiral symmetry breaking phases, Phys. Rev. D 82, 054009 (2010), arXiv:1007.1397 [hep-ph]

[107] R. Rapp, E. V. Shuryak, and I. Zahed, A Chiral crystal in cold QCD matter at intermediate densities?, Phys. Rev. D 63, 034008 (2001), arXiv:hep-ph/0008207

[108] E. Nakano and T. Tatsumi, Chiral symmetry and density wave in quark matter, Phys. Rev. D 71, 114006 (2005), arXiv:hep-ph/0411350

[109] T. Kojo, R. D. Pisarski, and A. M. Tsvelik, Covering the Fermi Surface with Patches of Quarkyonic Chiral Spirals, Phys. Rev. D 82, 074015 (2010), arXiv:1007.0248 [hep-ph].

[110] T. Kojo, Y. Hidaka, K. Fukushima, L. D. McLerran, and R. D. Pisarski, Interweaving Chiral Spirals, Nucl. Phys. A 875, 94 (2012), arXiv:1107.2124 [hep-ph].

[111] R. D. Pisarski, V. V. Skokov, and A. M. Tsvelik, Fluctuations in cool quark matter and the phase diagram of Quantum Chromodynamics, Phys. Rev. D 99, 074025 (2019), arXiv:1801.08156 [hep-ph].

[112] R. D. Pisarski, A. M. Tsvelik, and S. Valgushev, How transverse thermal fluctuations disorder a condensate of chiral spirals into a quantum spin liquid, Phys. Rev. D 102, 016015 (2020), arXiv:2005.10259 [hep-ph]

[113] Y.-L. Ma and M. Rho, Recent progress on dense nuclear matter in skyrmion approaches, Sci. China Phys. Mech. Astron. 60, 032001 (2017), arXiv:1612.06600 [nucl-th].

[114] D. Blaschke, T. Klahn, and D. N. Voskresensky, Diquark condensates and compact star cooling, Astrophys. J. 533, 406 (2000), arXiv:astro-ph/9908334.

[115] H. Grigorian, D. Blaschke, and D. Voskresensky, Cooling of neutron stars with color superconducting quark cores, Phys. Rev. C 71, 045801 (2005), arXiv:astro$\mathrm{ph} / 0411619$

[116] A. Cumming, E. F. Brown, F. J. Fattoyev, C. J. Horowitz, D. Page, and S. Reddy, A lower limit on the heat capacity of the neutron star core, Phys. Rev. C 95, 025806 (2017), arXiv:1608.07532 [astro-ph.HE]

[117] H.-X. Chen, V. Dmitrasinovic, and A. Hosaka, Baryon fields with $\mathrm{U}(\mathrm{L})(3) \mathrm{X} \mathrm{U}(\mathrm{R})(3)$ chiral symmetry II: Axial currents of nucleons and hyperons, Phys. Rev. D 81, 054002 (2010), arXiv:0912.4338 [hep-ph].

[118] H.-X. Chen, V. Dmitrasinovic, and A. Hosaka, Baryon Fields with $U_{L}(3)$ times $U_{R}(3)$ Chiral Symmetry III: Interactions with Chiral $(3, \overline{3})+(\overline{3}, 3)$ Spinless Mesons, Phys. Rev. D 83, 014015 (2011), arXiv:1009.2422 [hepph]. 
[119] H.-X. Chen, V. Dmitrasinovic, and A. Hosaka, mathrmBaryonswith $U_{L}(3) \times U_{R}(3)$ Chiral Symmetry IV: Interactions with Chiral $(8,1) \oplus(1,8)$ Vector and Axial-vector Mesons and Anomalous Magnetic Moments, Phys. Rev. C 85, 055205 (2012), arXiv:1109.3130 [hep-ph]

[120] H. Nishihara and M. Harada, Extended GoldbergerTreiman relation in a three-flavor parity doublet model, Phys. Rev. D 92, 054022 (2015), arXiv:1506.07956 [hepph]

[121] A. Motornenko, J. Steinheimer, V. Vovchenko, S. Schramm, and H. Stoecker, Equation of state for hot QCD and compact stars from a mean field approach, Phys. Rev. C 101, 034904 (2020), arXiv:1905.00866 [hep-ph],

[122] T. Kojo, D. Hou, J. Okafor, and H. Togashi, Phenomenological QCD equations of state for neutron star dynamics: Nuclear-2SC continuity and evolving effective couplings, Phys. Rev. D 104, 063036 (2021) arXiv:2012.01650 [astro-ph.HE].
[123] K. Fukushima, T. Kojo, and W. Weise, Hard-core deconfinement and soft-surface delocalization from nuclear to quark matter, Phys. Rev. D 102, 096017 (2020), arXiv:2008.08436 [hep-ph],

[124] L. McLerran and S. Reddy, Quarkyonic Matter and Neutron Stars, Phys. Rev. Lett. 122, 122701 (2019), arXiv:1811.12503 [nucl-th]

[125] K. S. Jeong, L. McLerran, and S. Sen, Dynamically generated momentum space shell structure of quarkyonic matter via an excluded volume model, Phys. Rev. C 101, 035201 (2020), arXiv:1908.04799 [nucl-th]

[126] T. Kojo, Stiffening of matter in quark-hadron continuity, Phys. Rev. D 104, 074005 (2021), arXiv:2106.06687 [nucl-th]

127] I. Tews, J. Carlson, S. Gandolfi, and S. Reddy, Constraining the speed of sound inside neutron stars with chiral effective field theory interactions and observations, Astrophys. J. 860, 149 (2018) arXiv:1801.01923 [nucl-th] 